\title{
Developments in target micro-Doppler signatures analysis: radar imaging, ultrasound and through-the-wall radar
}

\author{
Carmine Clemente $^{1 *}$, Alessio Balleri ${ }^{2}$, Karl Woodbridge $^{3}$ and John J Soraghan ${ }^{1}$
}

\begin{abstract}
Target motions, other than the main bulk translation of the target, induce Doppler modulations around the main Doppler shift that form what is commonly called a target micro-Doppler signature. Radar micro-Doppler signatures are generally both target and action specific and hence can be used to classify and recognise targets as well as to identify possible threats. In recent years, research into the use of micro-Doppler signatures for target classification to address many defence and security challenges has been of increasing interest. In this article, we present a review of the work published in the last 10 years on emerging applications of radar target analysis using micro-Doppler signatures. Specifically we review micro-Doppler target signatures in bistatic SAR and ISAR, through-the-wall radar and ultrasound radar. This article has been compiled to provide radar practitioners with a unique reference source covering the latest developments in micro-Doppler analysis, extraction and mitigation techniques. The article shows that this research area is highly active and fast moving and demonstrates that micro-Doppler techniques can provide important solutions to many radar target classification challenges.
\end{abstract}

\section{Introduction}

Moving targets illuminated by a radar system contain frequency modulations caused by the time-varying delay occurring between the target and the sensor. The main bulk translation of the target towards or away from the sensor induces a frequency or Doppler shift of the echo as a result of the well-known Doppler effect. However, the target can contain parts that can have additional movements. These can contribute frequency modulations around the main Doppler shift that are commonly referred to as micro-Doppler (m-D) modulations. Chen $[1,2]$ modelled the radar $\mathrm{m}-\mathrm{D}$ phenomenon and simulated $\mathrm{m}-\mathrm{D}$ signatures for various targets, such as rotating cylinders, vibrating scatterers and personnel targets. The authors also showed an effective tool in extracting the m-D signature is the time-frequency analysis of the received signal, leading to additional information on the target that can be used for classification and recognition. Micro-

\footnotetext{
${ }^{*}$ Correspondence: carmine.clemente@strath.ac.uk

${ }^{1}$ CeSIP, Department of Electronic and Electrical Engineering, University Of

Strathclyde, Glasgow, G1 1XW, UK

Full list of author information is available at the end of the article
}

Doppler can be regarded as a unique signature of the target that provides additional information about the target that is complementary to existing methods for target recognition. Specific applications include the recognition of space, air and ground targets. For example, the m-D effect can be used to identify specific types of vehicles and determine their movement and the speed of their engines. Vibrations generated by a vehicle engine can be detected by radar signals returned from the surface of the vehicle. For example, from $\mathrm{m}$-D modulations in the engine vibration signal, one can distinguish whether it is a gas turbine engine of a tank or the diesel engine of a bus. Another application is the use of $\mathrm{m}$-D signature for human identification making possible the identification of humans on different weather or light conditions. In particular, specific components of $\mathrm{m}-\mathrm{D}$ gait signature can be related to parts of the body for identification purposes [3]. Recently effective signature extraction techniques have been developed and tested on real data [4-11] providing features leading to classification results with a high level of confidence. These results could probably be improved if a multistatic m-D signature is used [12] where the self occlusion problem of the target is avoided.

\section{Springer}

(c) 2013 Clemente et al: licensee Springer. This is an Open Access article distributed under the terms of the Creative Commons Attribution License (http://creativecommons.org/licenses/by/2.0), which permits unrestricted use, distribution, and reproduction in any medium, provided the original work is properly cited. 
In this article, we review recent advances in radar based $\mathrm{m}-\mathrm{D}$ analysis over the last decade from radar imaging systems and emerging radar techniques. Our attention will focus on four fields that provide interesting results exploiting the m-D features: synthetic aperture radar, inverse synthetic aperture radar, ultrasound and through wall radar.

The remainder of the article is organised as follows. In Section 2, the basic concepts of micro-Doppler from a radar system are introduced. Section 3 is the review Section. Subsection 3.1 introduces the m-D effect studies for radar imaging platform, monostatic SAR, bistatic SAR and Inverse SAR. The exploitations of $\mathrm{m}$-D signatures proposed for ultrasound radar systems are described in Subsection 3.2 while Subsection 3.3 describes the ongoing research made in the field of the through the wall radar opening to the opportunity to extract $\mathrm{m}$-D also in the presence of obstacles.

\section{Micro-Doppler effect in radar}

The mathematics of the micro-Doppler effect from radar can be derived from introducing micro-motion to the conventional Doppler analysis. In this section the basics of the micro-Doppler effect are introduced. This is important for the understanding and the derivation of the microDoppler effects in more complex and realistic cases. In Figure 1 the geometry used to analyse the micro-Doppler induced by a vibrating target is shown [2]. The target located in $P$ vibrates with frequency $f_{v}$ and displacement $D_{v}$, thus having a displacement function of the kind $D(t)=D_{v} \sin \left(2 \pi f_{v} t\right) \cos \beta \cos \alpha_{p}$ (assuming $\alpha=0$ and $\beta_{p}=0$ ) [2]. Letting $R_{0}$ be the distance between the radar and the target initial position $O$ then the range function varies with time due to the target micro-motion $R(t)=$ $R_{0}+D(t)$. The radar received signal becomes

$$
\begin{aligned}
s(t) & =\rho \exp \left\{j\left[2 \pi f_{0} t+4 \pi \frac{R(t)}{\lambda}\right]\right\} \\
& =\rho \exp \left\{j\left[2 \pi f_{0} t+\Phi(t)\right]\right\},
\end{aligned}
$$

where $\rho$ is the backscattering coefficient $f_{0}$ is the carrier frequency and $\lambda$ is the carrier wavelength. Substituting the $R(t)$ in (1) the received signal can be expressed as:

$$
\begin{aligned}
s(t)= & \rho \exp \left\{j \frac{4 \pi R_{0}}{\lambda}\right\} \\
& \exp \left\{j 2 \pi f_{0} t+D_{\nu} \sin \left(w_{v} t\right) \cos \beta \cos \alpha_{p} 4 \pi / \lambda\right\},
\end{aligned}
$$

where $w_{v}=2 \pi f_{v}$. From (2), the derivative of the second phase term leads to the expression of the micro-Doppler shift.

$$
f_{m D}=\frac{w_{v} D_{v}}{\pi \lambda} \cos \beta \cos \alpha_{p} \cos \left(w_{v} t\right)
$$

Figure 2a,b show the simulation results for a $10 \mathrm{GHz}$ radar with a PRF of $2000 \mathrm{~Hz}$. Given $D_{v}=0.01 \mathrm{~m}, f_{v}=$ $2 \mathrm{~Hz}, \alpha_{p}=30^{\circ}, \beta_{p}=30^{\circ}$, and the center of the vibration at $(U=1000 \mathrm{~m}, V=5000 \mathrm{~m}, W=5000 \mathrm{~m})$. It is observed that the theoretical result, Figure 2a, of the micro-Doppler modulation is identical to the simulated results Figure 2b [2].

From this introduction to radar micro-Doppler it is evident how the ability to extract such an information from the returned signal can play an important role in modern automatic target recognition systems. In the following sections, recent advances in the analysis of micro-Doppler signatures from specific radar platforms are reviewed.

\section{Review}

\subsection{Micro-Doppler in radar imaging systems}

Radar imaging techniques such as synthetic aperture radar (SAR), inverse synthetic aperture radar (ISAR) and bistatic synthetic aperture radar (BSAR) are well established and useful techniques to acquire high-resolution

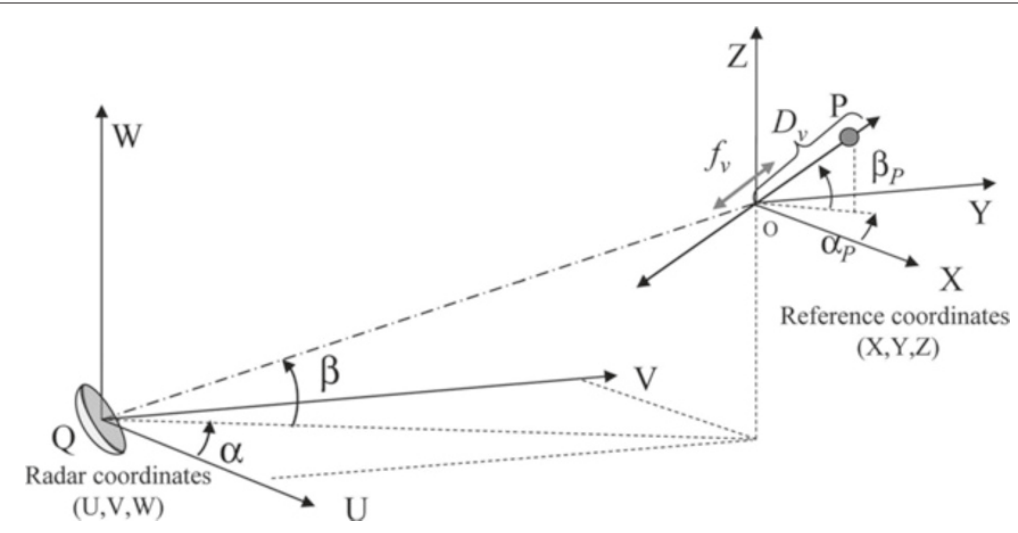

Figure $1 \mathrm{Geometry}$ for the radar and a vibrating point target [2]. 

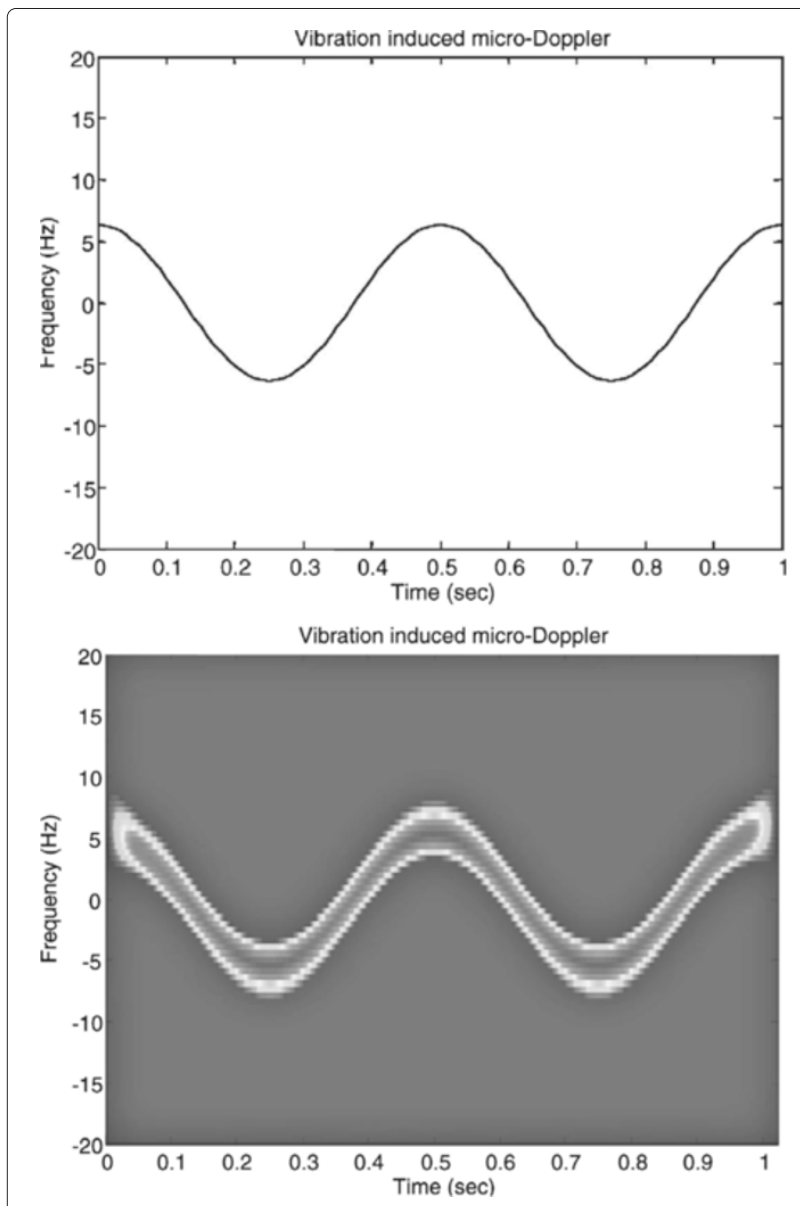

Figure 2 Theoretical and (Bottom) time-frequency representation of the micro-Doppler from a vibrating point target [2].

images of an area of interest from both airborne or space borne sensors. The amount of information provided from these systems is extremely high and its exploitation is still a growing field of research. For example, automatic target recognition based on the reflectivity images is a field of research with great interest and presenting many signal processing and design challenges [13].

The extraction of $\mathrm{m}-\mathrm{D}$ signatures from radar imaging systems for target classification purposes is an emerging technique. The research in this field provided interesting developments and results in the last years. This section will review the advances in the exploitation of the radar imaging platform for $\mathrm{m}-\mathrm{D}$ analysis, showing that from this kind of sensors a significant advantage can be taken if the $\mathrm{m}-\mathrm{D}$ information can be extracted.

\subsubsection{Synthetic aperture radar}

Synthetic aperture radar provides high resolution images of static ground scenes. The observed scenes may contain moving targets. As a result the processed image will change depending on the kind of target motion. Vibrating and rotating targets are two canonical cases that are often present within a scene. Rotating radar search antennas, wind turbines and vehicles that vibrate due to the engine are typical targets that exhibit micro-motions and related $\mathrm{m}-\mathrm{D}$ characteristics. It is often possible to observe these micro-motions from a SAR. The analysis of the m-D features has been modelled and experimentally characterised for different type of targets, configurations and working frequencies over the last decade.

In [14], a derived signal model of the received baseband SAR signal from a target with $\mathrm{m}-\mathrm{D}$ frequency $f_{m D}$ was shown to be:

$$
s_{b}(t, T)=s(t, T) e^{-j\left(2 \pi f_{m D}(T)\right) t},
$$

where $s(t, T)$ is the echo from a stationary target, $t$ is the fast time and $T$ is the slow time. For a vibrating target with vibrating amplitude $A_{v}$ and vibrating frequency $w_{v}$, the resulting $m-D$ is:

$$
f_{m D_{\mathrm{vib}}(T)}=\frac{-2 A_{v} w_{v} \sin \left(w_{v} T\right)}{\lambda_{c}},
$$

where $\lambda_{c}$ is the carrier wavelength $[14,15]$. This result is principally due to the cosine behaviour of the target in the radial direction. For a rotating target the motion presents a cosine and a sine component in quadrature leading to the following m-D frequency shift:

$$
\begin{aligned}
f_{m D_{\text {rot }}(T)=\frac{2 r w_{r}}{\lambda_{c}}} & \left(\sin \left(w_{r} T\right) \cos (\Theta(T))\right. \\
& +\cos \left(w_{r} T\right) \sin (\Theta(T)),
\end{aligned}
$$

where $r$ is the rotating radius, $w_{r}$ is the rotating frequency and $\Theta(T)$ is the instantaneous aspect angle [14].

The case of an oscillating point target was investigated by Sparr and Krane [15]. They showed that the m-D contribution for such targets in monostatic SAR may be seen as a sinusoidal phase modulation of the SAR azimuth phase history. In [15], the analysis method is also investigated, using quadratic time-frequency methods obtaining high time-frequency resolution representations. Real APY-6 SAR datasets were used to test the adaptive optimal Kernel (AOK) to analyse the ground target oscillations. AOK was demonstrated to be effective in dealing with the problem of the selection of the sufficiently short time window to resolve properly the time frequency distribution of oscillating targets. In Figure 3, the experimental result using AOK analysis of a reflector with $2.0 \mathrm{~Hz}$ vibrating frequency is shown [15]. The m-D signature varies between 70 and $85 \mathrm{~Hz}$ with a $2.5 \mathrm{~Hz}$ oscillation peak-topeak and a period of $0.5 \mathrm{~s}$. This information introduces a modulation frequency of $2.0 \mathrm{~Hz}$ in accordance with the ground truth. However, in the plot the resulting amplitude of the vibration is $1.5 \mathrm{~mm}$ while the ground truth was of $8 \mathrm{~mm}$. In [15], this error is not analysed in depth. 


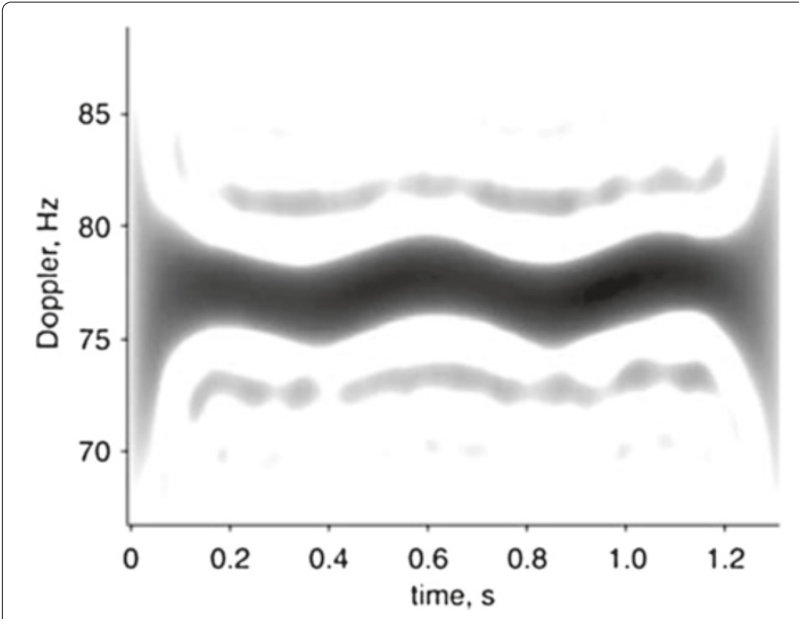

Figure 3 Time frequency signature of oscillating reflector [15] analysed using the AOK method showing a feature oscillating between 70 and $85 \mathrm{~Hz}$ with a $2.5 \mathrm{~Hz}$ oscillation peak-to-peak with a $0.5 \mathrm{~s}$ of periods. These values links to the ground truth.

Figure 4a shows the result obtained by using a window made of 256 samples, the same length used to obtain the result in Figure 3. Figure $4 \mathrm{~b}$ shows the result obtained with a window size of 16 samples. These results show that a longer window length results in a more-or-less stationary spectrum (which can be quite noisy), whereas a smaller window length produces a single instantaneous frequency with complicated time variations. From the results a modulation period of $0.5 \mathrm{~s}$ with a peak-to-peak deviation of $120 \mathrm{~Hz}$ has been estimated. These values correspond to a modulation frequency of $20 \mathrm{~Hz}$ with an amplitude of $7 \mathrm{~mm}$, in agreement with the ground truth. This work was one of the first m-D analysis of real data showing the feasibility of detecting m-D signatures to be exploited for target recognition purposes.

Silverstein and Hawkins [16] analytically derived the $\mathrm{m}-\mathrm{D}$ signature of rotating objects in Synthetic Aperture Radar and highlighted differences with the vibrating case. They pointed out that the order required in the approximation of the expansion of the phase history needed to be greater than first order in the case of a rotation, while for the vibrating case a first order approximation was sufficient. The main reason was that in a rotational target the motion was large compared to the wavelength so a numerical analysis was required.

In [17], Sparr presented the results of an experiment with a target moved along a trajectory in the azimuth direction, with oscillations superimposed in the range direction. Time frequency analysis methods were applied to the obtained datasets to show that $\mathrm{m}-\mathrm{D}$ signatures can be exploited to distinguish targets with micro-motions from stationary targets. Experimental results are shown in Figure 5. Figure 5a shows the time-frequency analysis

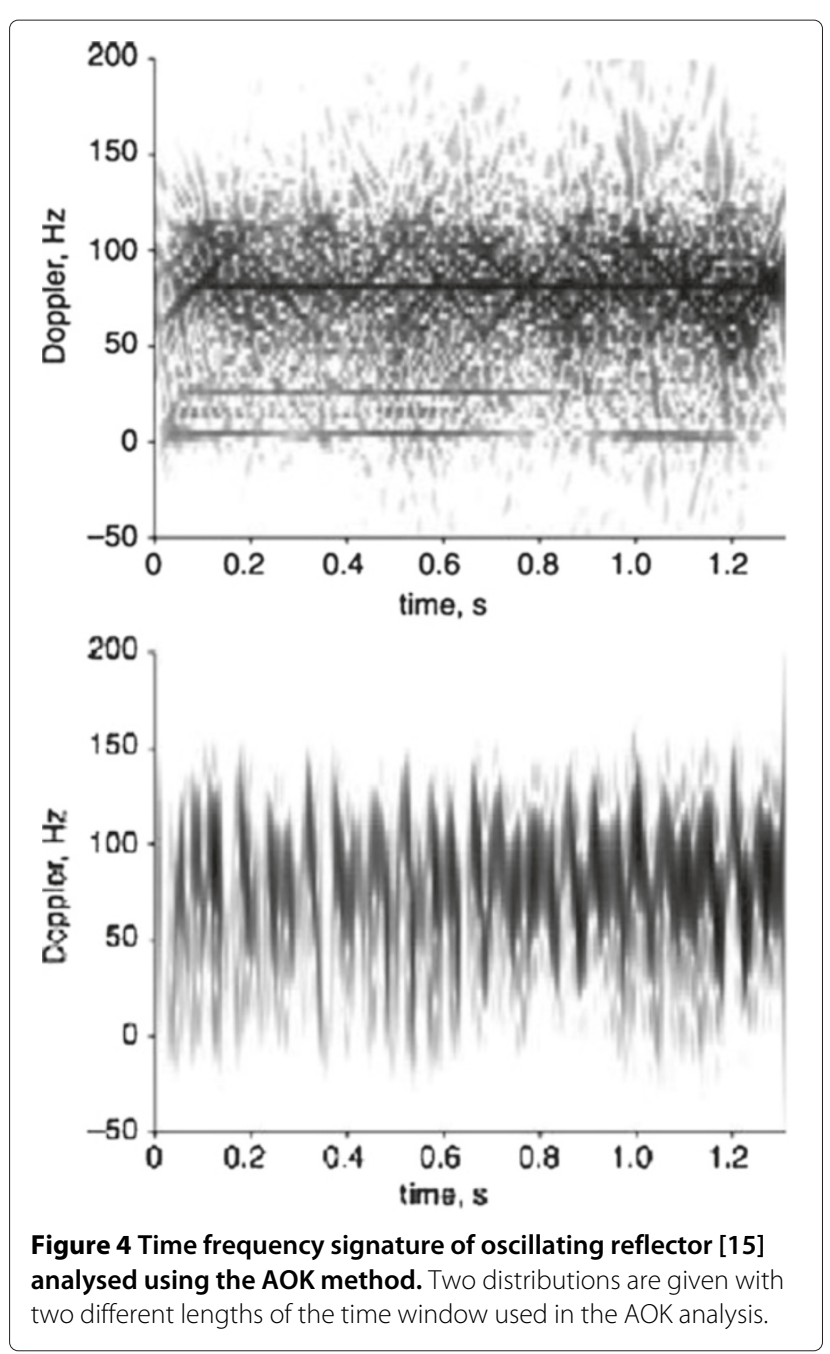

results of a moving target with a radial micro-motion. In this figure the sinusoidal-like signature due to the micromotion is evident. Figure $5 \mathrm{~b}$ shows the signature of a building. In this result only the stationary component due to the edge of the building and that of the multipath component can be seen. The m-D effect from rotating and vibrating targets in millimetre-Wave SAR was analysed in [14]. The very small wavelength facilitates the observation of very small vibrations such as those of a truck. Results for both simulated and real vibrating and rotating targets were presented in [14]. The smoothed pseudo Wigner Ville Distribution (PWVD) was used to provide a high resolution time-frequency representation. For real data, where the presence of noise affects the signal, the Gabor distribution was preferred. In Figure 6 the WVD of experimental data [14] is shown. The dataset consists of data acquired over two trucks; Figure 6a refers to the static truck, while Figure $6 \mathrm{~b}$ refers to the case of the truck with the engine switched on vibrating at $32 \mathrm{~Hz}$ and with a static second harmonic at $64 \mathrm{~Hz}$. The plot of the static 

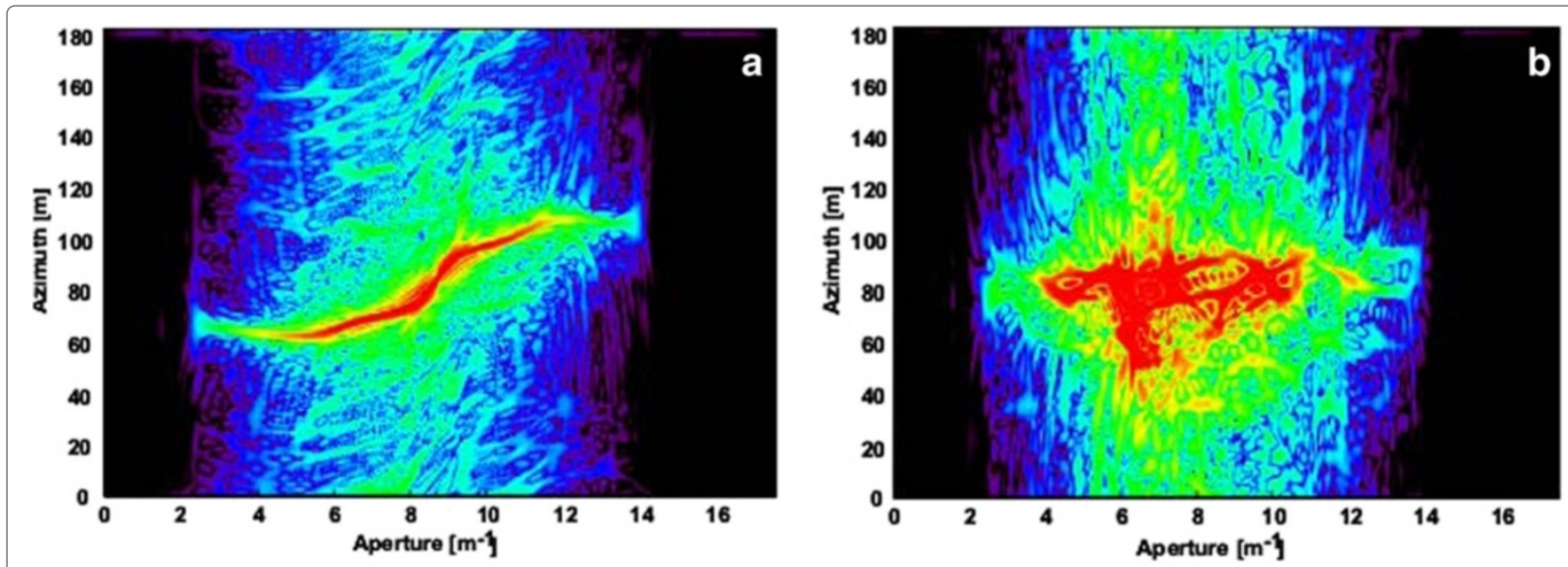

Figure 5 Time frequency analysis of (a) the moving target with micro-motions and (b) a stationary building from [17].

truck shows two very prominent areas around $\pm 150 \mathrm{~Hz}$. In [14], this effect was justified by the space between the cab and the trailer of the truck which acted as corner reflector. In this case no pattern can be clearly recognised other than fluctuations due to the truck geometry. For the vibrating truck, Figure $6 \mathrm{~b}$, a m-D pattern appears where a periodic signature with a period of 0.03 s corresponding to the $32 \mathrm{~Hz}$ of vibration of the truck. However, this feature is difficult to extract due to the strong return from the truck geometry.

The effect on SAR images has been also analysed [1,14]. In this case if the micro-motion appears in the azimuth direction then this will cause smearing of the target. If the phase modulation is in the range direction then this causes an effect in SAR images known as paired echoes. The result of this effect will be the appearance of ghost targets in the cross-range direction on both sides of the original target. The target vibration can then introduce an infinite series of paired echoes $k$ because, when considering (5), the received signal (4) may be expressed as a series of expansion of Bessel functions of the first kind of order $k[1,14]$ :

$$
J_{k}(B)=\frac{1}{2 \pi} \int_{-\pi}^{\pi} e^{j\left(B \sin \left(w_{v} T\right)-k w_{v} T\right)} d\left(w_{v} T\right),
$$

where $B=A_{v}\left(4 \pi / \lambda_{c}\right)$ and $J_{k}(B)$ decreases with increasing $k$. Thus the received signal can be written as:

$$
s_{b}(t, T)=s(t, T) \sum_{k=-\infty}^{\infty} J_{k}(B) e^{j k w_{v} T} .
$$

These ghost targets can appear stronger than the original target thus leading to incorrect interpretations of the focussed image that can mask other targets of interest.

From [14], focussed simulated $94 \mathrm{GHz}$ SAR data are presented in Figure 7. In Figure 7a, the simulated targets are vibrating at $30 \mathrm{~Hz}$ while in Figure $7 \mathrm{~b}$ they are vibrating at $50 \mathrm{~Hz}$. Vibration amplitudes from left to right are 10, 1 , $0.1,0.01$ and $0.001 \mathrm{~mm}$ for both cases. Targets with very
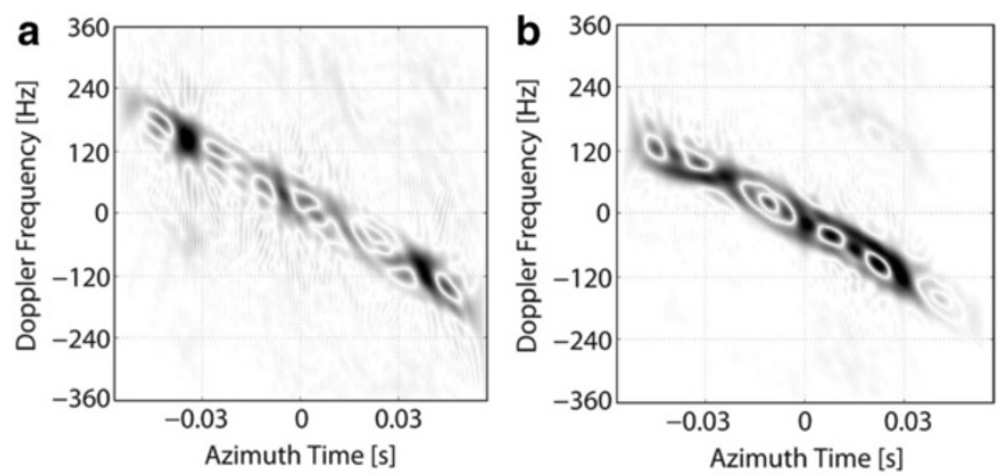

Figure 6 Time-frequency representation (WVD) of the Doppler spectrum for (a) a static truck and (b) a vibrating truck [14]. 

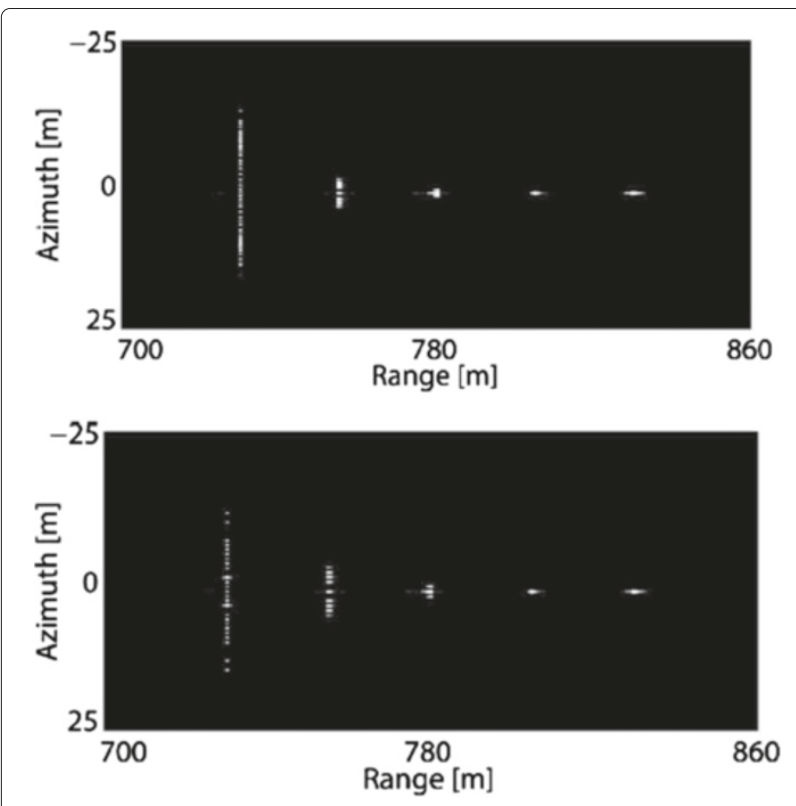

Figure 7 Focused 94-GHz SAR images, each with five simulated targets vibrating at (Top) $30 \mathrm{~Hz}$ and (Bottom) $50 \mathrm{~Hz}$. Vibration amplitudes from left to right are 10, 1, 0.1, 0.01, and $0.001 \mathrm{~mm}$ for both images [14].

large vibrations clearly show multiple and very densely spaced paired echoes in accordance with (8).

The most relevant recent work on monostatic SAR m-D was presented in [18]. In this paper, it was pointed out that in most of the previous work the targets were assumed to be point scatterers and that this assumption may not always agree with reality. The effect of micro-motions using distributed scatterers and localized scatterer model was addressed in this paper. Also more accurate and complex micro-motion models are introduced for rotating, vibrating sinusoidal moving and rocking targets, providing a good set of tools for the analysis in SAR. Two novel models for the phase history of the received echoes described in [18] includes: (1) A sawtooth expansion for a more accurate model of the phase modulation introduced by micro-motions and (2) a generalized paired echoes principle (PEP) to model the effect on the focussed image the general cases like a rocking ship.

An analysis of the effect on focussed images was also reported in [18] with the distinction of different micromotions and their amplitude and frequency characteristics. The effect on different imaging algorithms was reported showing that the focussing algorithm plays a key rule in the final effect on the image. The loss in azimuth resolution and the GMTI and MTI problems introduced by micro-motions was analysed. These aspects are not of direct interest in this review and will not be further considered.
All the previously presented papers focussed on the demonstration of the models of micro-Doppler signatures from SAR. For this reason all experiments were carried in cases of high signal to clutter ratio (SCR). This assumption often is not valid and strong clutter can bury the useful micro-Doppler signature.

To deal with this problem an extraction technique is required. In [19], the Singular Spectrum Analysis was applied to simulated SAR data. The applied procedure starts from the range compressed data, then selecting the range gate containing the scatterer with micro motions. Depending on the analysed configuration a range cell migration correction step can be performed before the extraction of the range gate. In order to align the signal within the time-frequency plane to obtain a correct visualization and positioning of the time-frequency distribution, the Doppler centroid and the azimuth frequency slope is removed from the signal before the SSA. After the selection of the singular components the resulting micro-Doppler signature can be visualized and used for target classification through the computation of a time frequency distribution.

The effectiveness of the technique was demonstrated in low SCR with K-distributed clutter that model correctly the case of sea clutter in SAR and bistatic SAR clutter.

\subsubsection{Bistatic SAR}

Of particular interest is the bistatic SAR case (BSAR). BSAR operates with a separate transmitter and receiver introducing new characteristic compared to traditional monostatic SAR systems. The bistatic configuration with a fixed receiver was considered in [20] wherein an expression for the bistatic $m$-D signature of vibrating targets was derived. The bistatic case shows a substantial difference from the traditional monostatic case. The bistatic m-D signature is influenced by the bistatic acquisition geometry. It was demonstrated that the bistatic configuration exhibits the advantage of a reduced minimum required pulse rate frequency (PRF) compared to that required in the monostatic case, reducing potential aliasing. The resulting $\mathrm{m}$-D shift obtained in a bistatic SAR is [20]

$$
f_{B m D_{\mathrm{vib}}(T)}=\frac{f_{m D_{\mathrm{vib}}(T) \rho}}{2},
$$

where $\rho$ is the bistatic factor including the geometry dependence $\rho=\sin \left(\Theta_{R}\right) \cos \left(\alpha_{R}\right)+\sin \left(\Theta_{T}\right) \cos \left(\alpha_{T}\right), \Theta_{R}$ and $\Theta_{T}$ represent the transmitter and the receiver depression angle while $\alpha_{R}$ and $\alpha_{T}$ represent the receiver and transmitter squint angle, respectively. The fact that monostatic and bistatic m-D signatures are related is useful when exploiting existing monostatic SAR m-D signatures in the generation of a bistatic SAR m-D signatures 
database for target classification for a given bistatic geometry without the necessity to perform further data acquisition. The amplitude of the vibration can be obtained from the measured maximum of the m-D signature as [20]

$$
A_{v}=\frac{\lambda_{c} \max \left(f_{M D}(\eta)\right)}{w_{v} \rho}
$$

Different acquisition geometries induce different m-D shifts and thus a good knowledge of the geometry is required in order to obtain the amplitude of the vibration. Figure 8a,b show the theoretical bistatic maximum of the m-D signature for a $94 \mathrm{GHz}$ and a $10 \mathrm{GHz}$ system respectively for a vibrating target with $w_{v}=20 \pi \mathrm{rad} / \mathrm{sec}$ and a vibration amplitude from $0.1 \mathrm{~mm}$ to $10 \mathrm{~mm}$. The amplitude of the $\mathrm{m}$-D signature depends also on the vibrating frequency $w_{v}$ as can be seen in (10).
Figures 9a,b show the theoretical bistatic maximum of the m-D signature with a $94 \mathrm{GHz}$ and a $10 \mathrm{GHz}$ system for a vibrating target with $1 \mathrm{~mm}$ of vibrating amplitude and with a vibrating frequency from 1 to $100 \mathrm{~Hz}$. For both cases the bistatic factor influences the amplitude of the $\mathrm{m}-\mathrm{D}$ signature. This factor is the sum of the conic angle of the transmitter and receiver and therefore is dependent on the BSAR geometry [20]. From both Figure 7a,b, the bistatic factor influences the amplitude of the m-D signature, when $\rho=2$ the curves assumes the maximum values and they coincide with the maximum micro-Doppler obtained in a monostatic case. This aspect must be considered because a certain amplitude of the m-D signature corresponds to a family of vibrating amplitudes. The correct amplitude can be obtained only with the knowledge of the bistatic factor. From (9) it is seen that the bistatic factor reduces the amplitude of the m-D signature compared
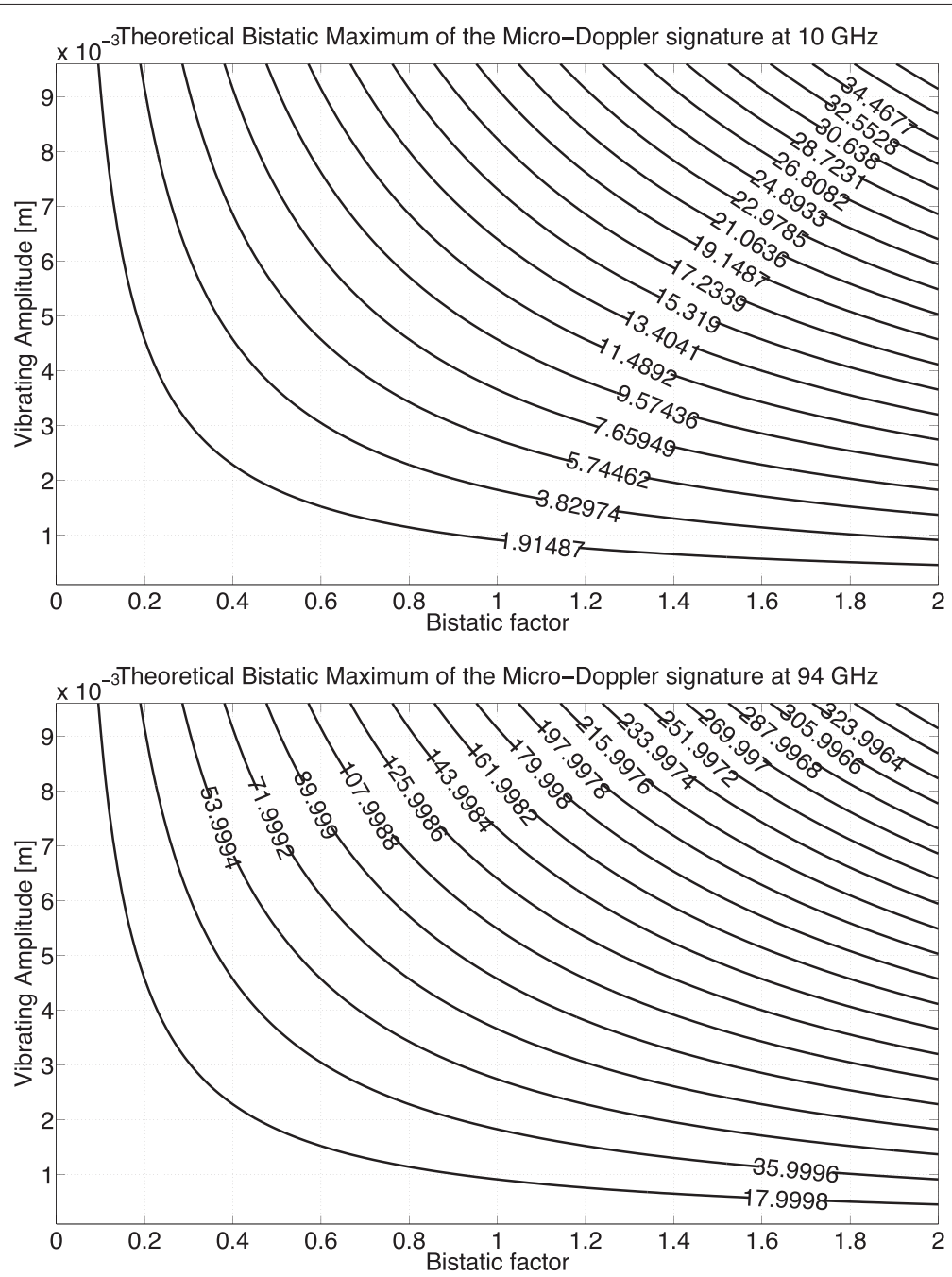

Figure 8 Maximum amplitude of the micro Doppler frequency for different bistatic configurations for a vibrating target with a vibrating frequency of $10 \mathrm{~Hz}$ and vibrating amplitudes from $0.1 \mathrm{~mm}$ to $10 \mathrm{~mm}$. (Top) micro Doppler for a $10 \mathrm{GHz}$ system (Bottom) micro Doppler for a $94 \mathrm{GHz}$ system. The micro Doppler is increasing with the increasing vibrating amplitude and bistatic factor [20]. 

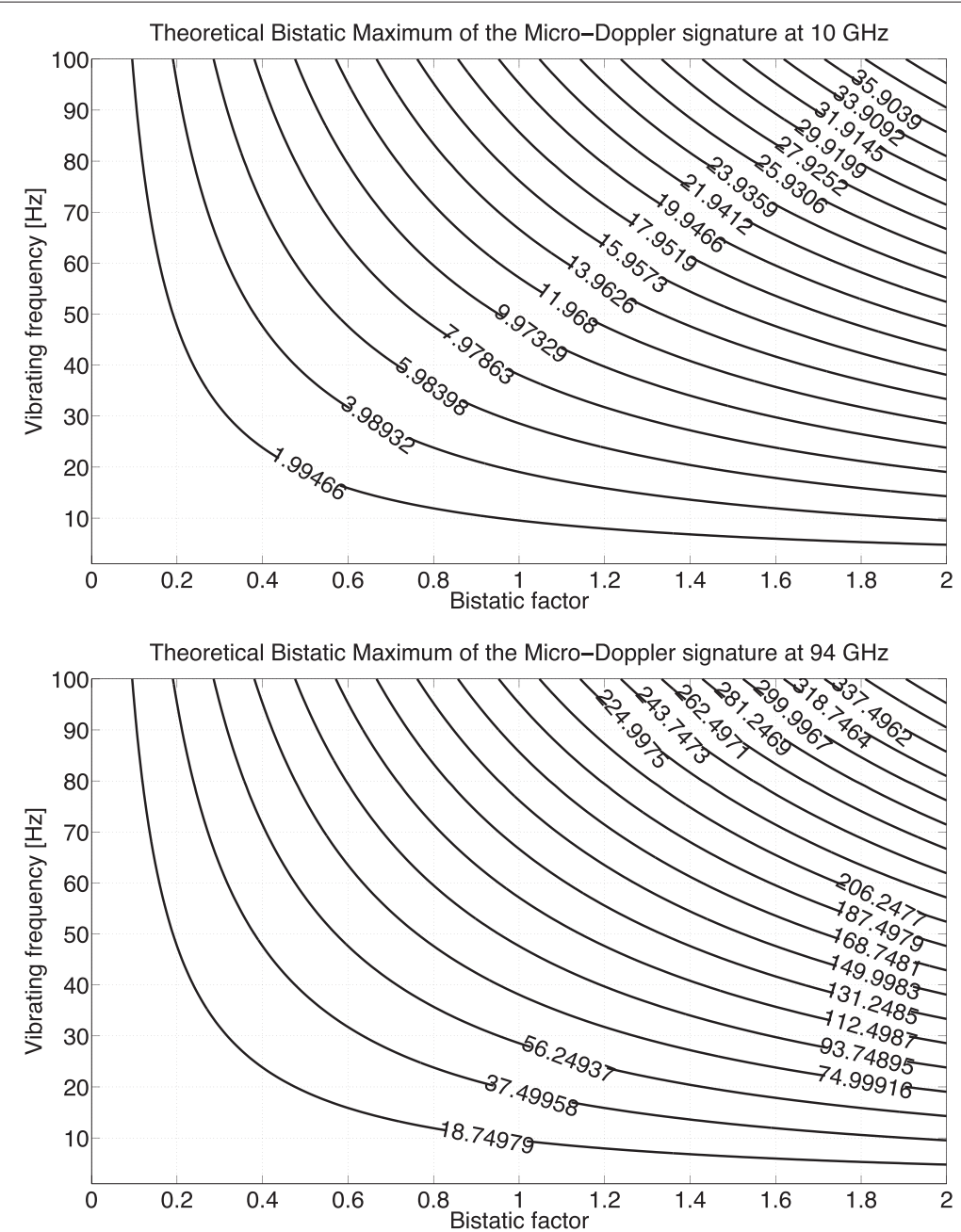

Figure 9 Maximum amplitude of the micro Doppler frequency for different bistatic configurations for a vibrating target with a vibrating amplitude of $1 \mathrm{~mm}$ and vibrating frequencies from 1 to $100 \mathrm{~Hz}$. (Top) micro Doppler for a $10 \mathrm{GHz}$ system (Bottom) micro Doppler for a $94 \mathrm{GHz}$ system. The micro Doppler is increasing with the increasing vibrating frequency and bistatic factor [20].

with that obtained for the same vibration in the monostatic case. This means that for vibrations introducing high Doppler shifts, running over the azimuth Doppler bandwidth in the monostatic case, the analysis of the signature can suffer from aliasing.

However, as the use of a bistatic configuration reduces the m-D amplitude it is possible to keep the signature inside the azimuth Doppler bandwidth. This means that it is possible to use a smaller minimum PRF to avoid aliasing in the analysis compared to the monostatic configuration.

As in the monostatic case the vibration introduces paired echoes in the resulting bistatic SAR image, the signal model can still be expanded into a series of Bessel functions of the first kind but with a different value of the argument of the Bessel functions depending on the bistatic factor. This introduces an acquisition geometry dependence of the effect on the focussed image.
In order to address the issues of micro-Doppler hiding in clutter and the absence of the micro-Doppler signature if the target is moving along the radial direction of the common monostatic SAR a displaced phase center array (DPCA) based technique was proposed in [21]. The authors proposed a configuration with a fixed receiver and a dual channel receiver separated by phase centres. The two channel information is exploited to cancel the stationary clutter and keep the incoherent signal due to the micro-motion of the target. In addition the authors exploited a Fractional Fourier Transform based estimation of the signal Doppler to correct the motion of the transmitting platform. However, two main limitations are present in this proposed technique: synchronization and modulation of the backscattered energy from the target with micro-motions. The authors assumed that all the synchronization issues for the proposed configuration 
are accurately corrected, however for the problem under analysis even a small error on the synchronization of the two channel signals with the receiver would make the extraction of the micro-Doppler ineffective. The second issue is the presence of a sinusoidal modulation of the amplitude of the energy of the micro-Doppler signature due to the DPCA processing. This would produce a weaker micro-Doppler signal that could be difficult to detect and exploited for classification purposes in a noisy environment.

\subsubsection{Inverse SAR}

In inverse synthetic aperture radar imaging the radar platform is stationary and the target is moving with the assumption of a quasi-constant linear motion. Other target motions will produce errors in the final focussed image. The Doppler resolution is inversely related to the total time of coherently processed pulses. If the duration of the coherent processing interval (CPI) is too long, then uncompensated motion could affect the image producing smearing in the Doppler dimension, affecting the Doppler resolution too.

Specific analysis and experiments about the analysis of wheels and pedestrian in ISAR was presented in [22]. The analysis first focussed on the model of the m-D effect from wheels of civil vehicles, demonstrating (with both simulated and real data) the possibility of extracting all the information required to correctly identify size, position, aspect angle and rotating frequency of a rotating wheel. The analysis then considered the case of the pedestrian, where an analysis of the features characterizing walking/running was reported. The paper demonstrated also the possibility to obtain information of the gait of the pedestrian using of time-frequency analysis on both simulated and real data.
In the last decade, however, the interest of the ISAR community moved from the analysis of the m-D signatures to the most challenging problem of the compensation of the micro-motions in order to obtain good focussed ISAR images. In [23], a good review of the m$\mathrm{D}$ analysis in ISAR was presented. Methods for analysing, visualizing, exploiting $\mathrm{m}-\mathrm{D}$ signatures are reviewed in conjunction with examples of models for rigid and nonrigid body motions. Finally, [23] introduces perspective in ISAR m-D exploitation.

The problem of extracting the modulation due to the micro-motion from the received signal has been addressed using various signal processing approaches. In [24], a Chirplet decomposition method to separate the rotating and vibrating micro-Doppler from the target body echo is presented. The Wigner-Ville distribution is used for the time-frequency analysis exploiting the specific properties of the Gauss-Chirplet, in particular the absence of cross-terms. Different Chirplet bases are used to project the received echo in different Chirplet domains obtaining the separation of the body echoes from the micro-motion echoes on both simulated and real data. Fulin and Mingyuan in [25] proposed the use of the Singular Spectrum Analysis (SSA) to separate the $\mathrm{m}$-D components from the rigid body components. SSA is used to decompose the original received signal in low frequency signal, quasi-periodic signals and noise. The SSA is applied to the range compressed signal. In this case the rigid body will be represented by a singular eigenvalue in the eigenvalue spectrum. This aspect is very helpful for the separation of the contributions. Results using the SSA based method from [25] are shown in Figures 10 and 11. It is clear how the micro-motion modulation affects the resulting focussed image, Figure 10a and how selecting the correct components it is possible to obtain a clean image, Figure 11d.
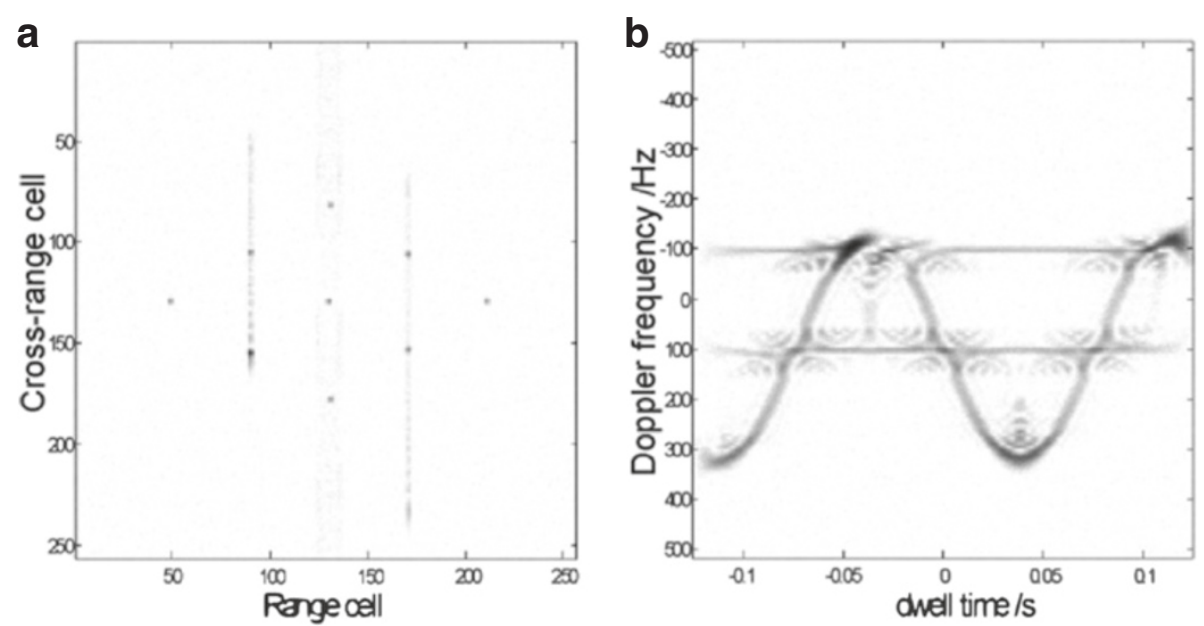

Figure 10 Analysed case. (a) Simulated ISAR image and (b) the time-frequency distribution of range cell 89 [25]. 
(a)

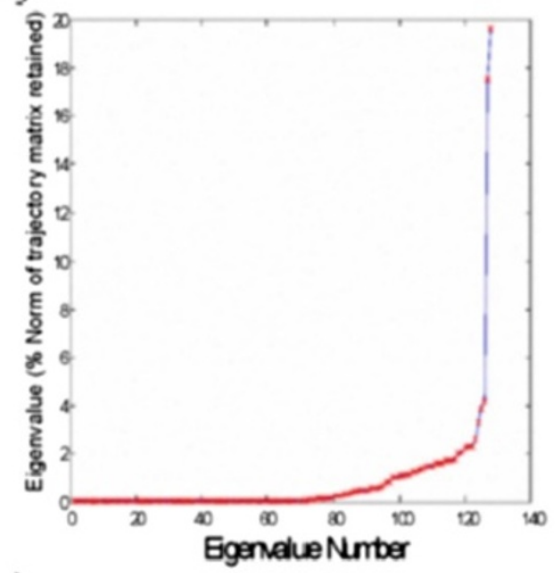

(c)

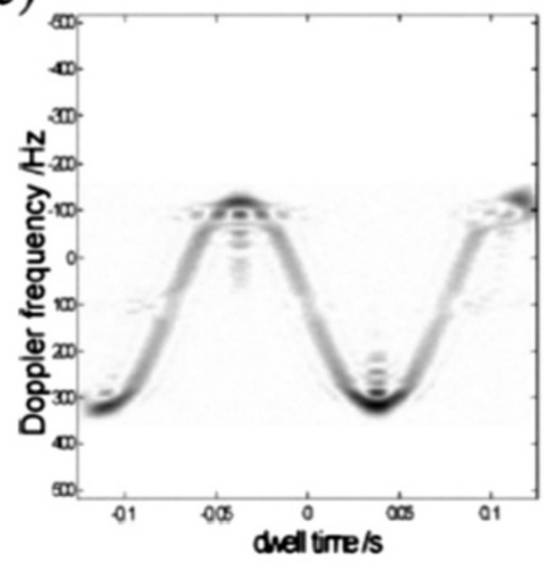

(b)

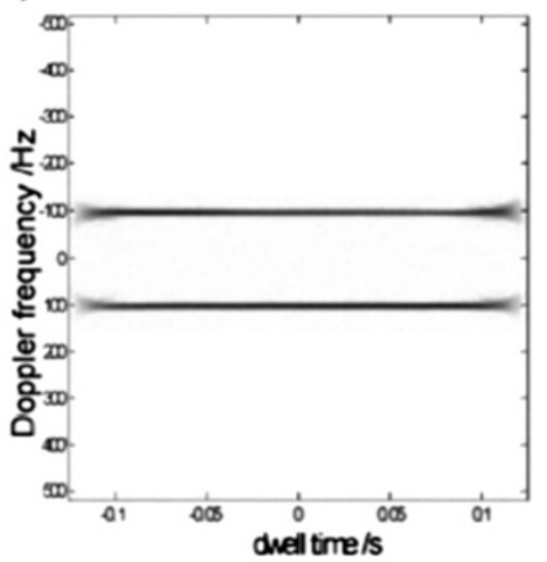

(d)

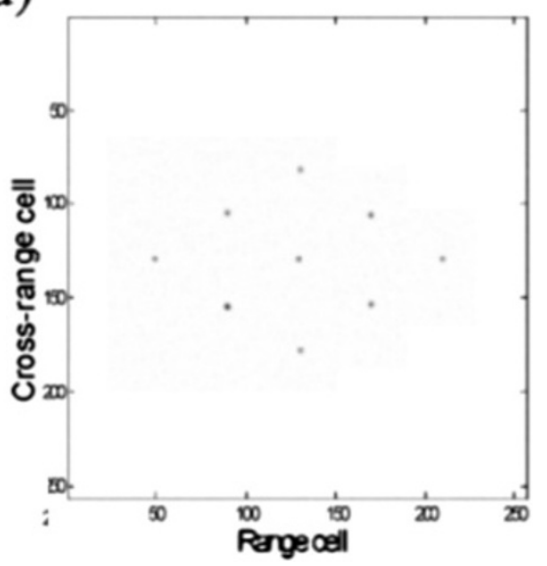

Figure 11 SSA results. (a) The singular spectrum of the $\mathbf{8 9}^{\text {th }}$ cell, (b) the time frequency distribution of the reconstruction signal, (c) the time-frequency distribution of the residual signal and (d) the cleaned ISAR image after the SSA [25].

The next part of this review section will focus on the imaging techniques used to mitigate the effect of micromotions. In [26], a novel imaging method of birds was proposed, wherein a technique to remove the flapping spectrogram from the data firstly by the variety of moving average values of the cross-correlation coefficients of the high-resolution range profiles, and then removing the flapping spectrogram. In addition genetic algorithms and minimum waveform entropy were used for phase compensation. The effectiveness of the technique was demonstrated by simulations.

A real time imaging technique is proposed in [27]. The proposed technique used a time-frequency s-method based on the relationship between the short time Fourier transform and the Wigner Distribution. The s-method was tested on simulated data of a Boing-717, a MIG-25, a Delta Wing and a Canadian Coast Guard Vessel providing good results in terms of motion compensation and computational efficiency.
The complex valued empirical mode decomposition (CEMD) was proposed in [28] to remove the micromotion due to rotating parts in a target. The CEMD is able to identify rotating intrinsic mode functions (IMFs) for this reason was selected as good technique to separate the different motion components. The technique was tested on both simulated and real datasets. In Figure 12 the results presented in [28] are reported. The results refer to the application of the CEMD based technique to real data of a AN-26 plane. Figure 12a shows the spectrogram of the echo in range cell 131. It can be seen that the $\mathrm{m}$-D occupies the whole spectrum due to the frequency modulation of turbo blades. Figure $12 \mathrm{~b}$ shows the Range Doppler image of the data before the CEMD processing, while in Figure 10c shows the same image after the CEMD processing. The last plot, Figure 12d shows the normalized autocorrelation coefficient for the $\mathrm{m}-\mathrm{D}$ in range cell 131, from this figure the rotation rate of $20 \mathrm{~Hz}$ can be estimated [28]. 


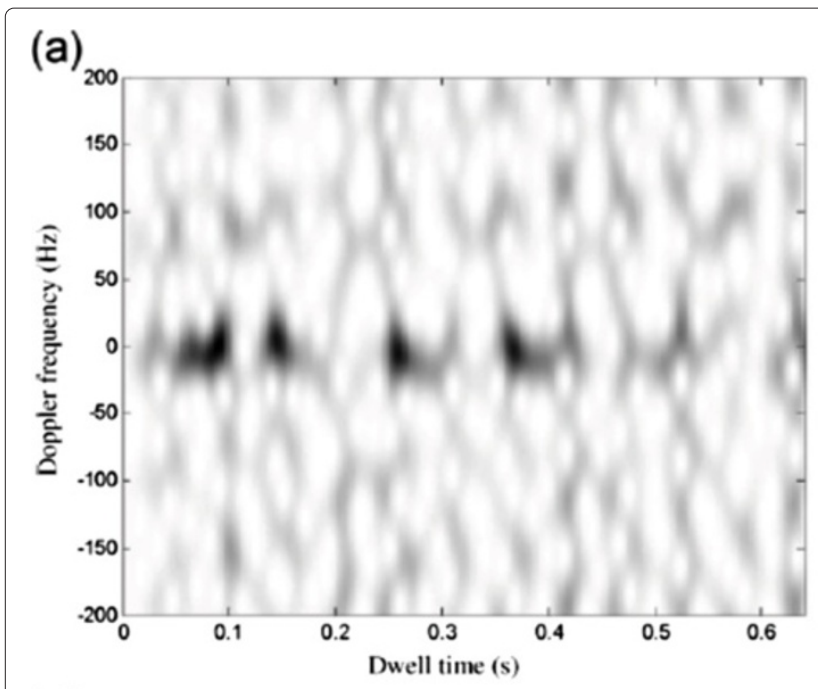

(c)

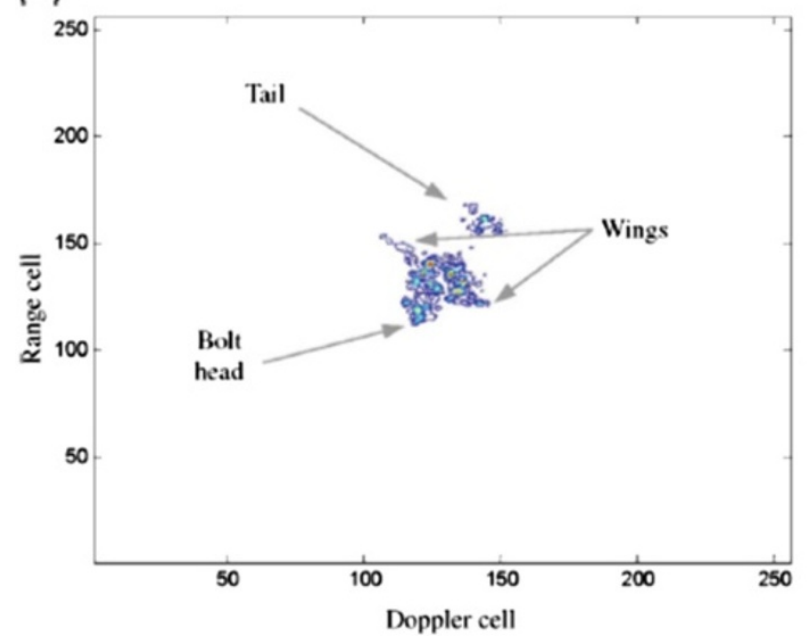

(b)

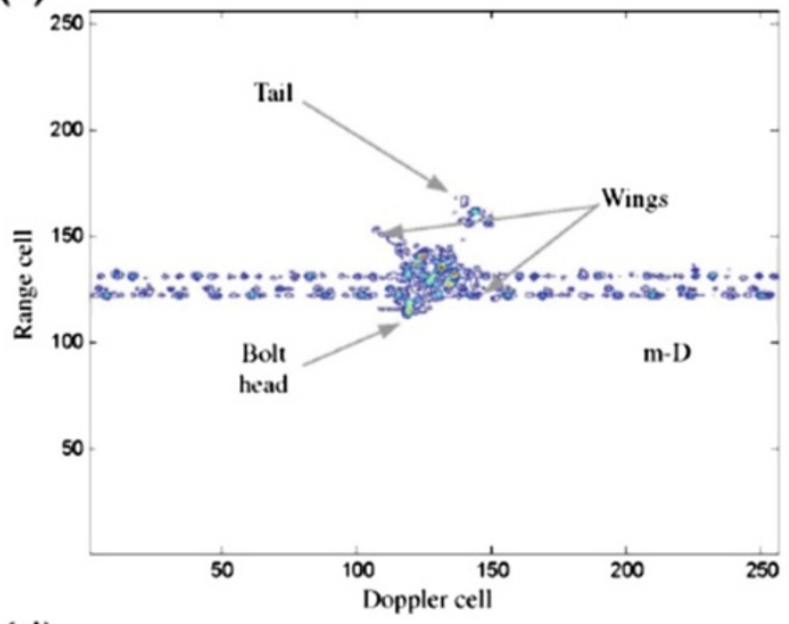

(d)

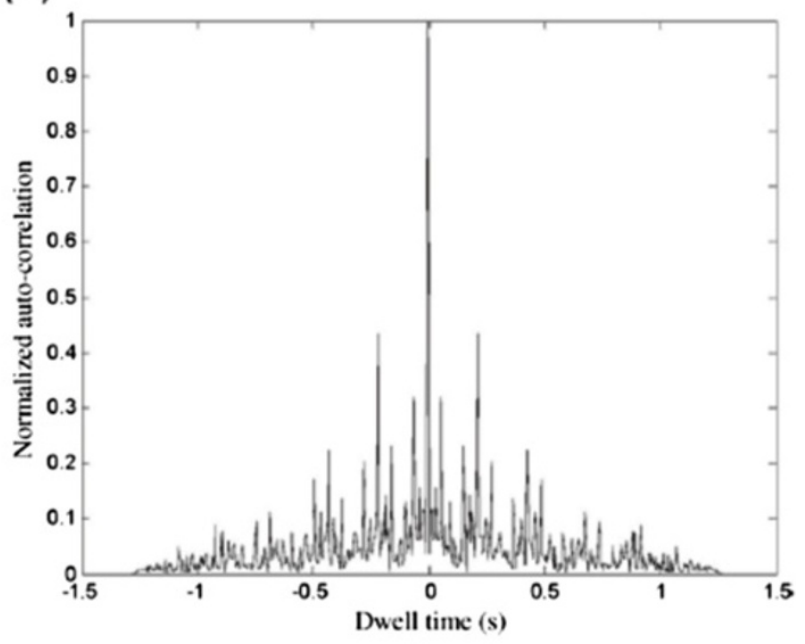

(b) Image of raw data. (c) Image of the main body. (d) Normalized

Figure 12 Image for measured data. (a) Spectrogram for range cell 131. autocorrelation of $\mathrm{m}-\mathrm{D}$ in range cell 131. [28].
In [29], another approach for ISAR imaging of targets with rotating parts was presented. The authors proposed a data-recording model for two-dimensional (2D) ISAR imaging of rotating parts. The the real-valued inverse Radon transform was then applied to image formation in the range-slow time domain. The rotating parts are imaged using complex-valued inverse Radon transform. The authors start from the assumption that for wideband radar, the $\mathrm{m}-\mathrm{D}$ signal induced by a rotating scatterer has sinusoidal modulus and phase in the range-slow time domain, which is equivalent to the Radon transform of its distribution function. Therefore, it can heuristically perform the inverse Radon transform to reconstruct the image. The authors then proposed two methods for ISAR imaging of rotating parts. In the range-slow time domain, the first method executes real-valued inverse Radon transform (RIRT) to echo modulus directly, while the second one applies complex-valued inverse Radon transform (CIRT) to the complex echoes. Because it performs coherent integration, the CIRT method achieves a higher spatial resolution than the RIRT method. The approach was tested on real AN-26 Plane data, which has a turbo on each side of the airframe. Figure 13a shows the range Doppler image obtained from the echoes where the $\mathrm{m}$-D strips due to the turbines can be observed. After the application of the m-D separation using chirplet decomposition the focussed image resulted to be less noisy as shown in Figure 13b-d show the results of the focussed turbines using the RIRT algorithm. The four blades can be easily identified proving the effectiveness of the approach [29].

The case of fast rotating targets was addressed in the technique proposed in [30]. In this paper a segmental Pseudo keystone transform (SPKT) was introduced to 

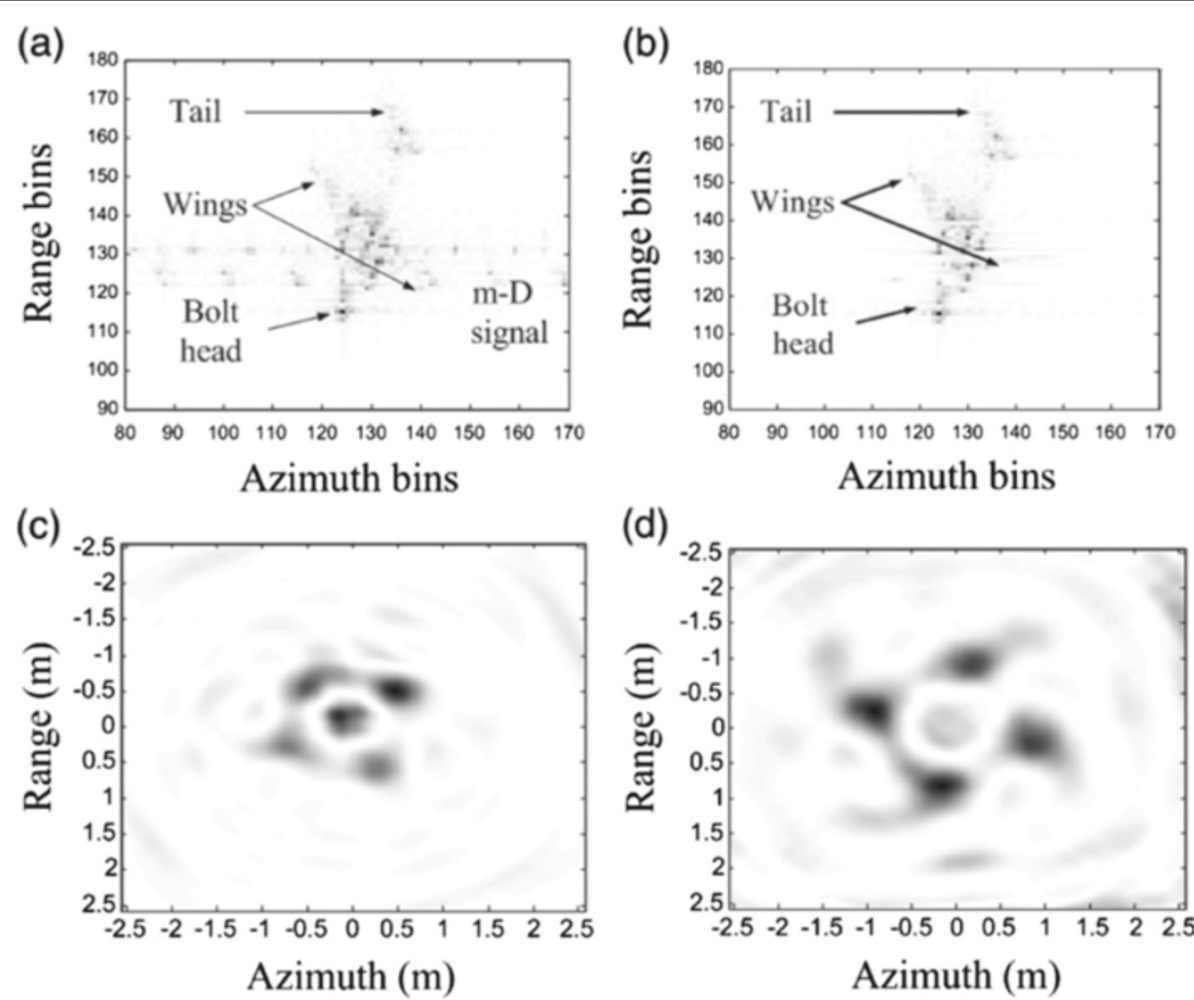

Figure 13 Image of AN-26 plane. (a) Image of the data with m-D. (b) Image of the data with the radon based approach. (c) Image of the first turbo. (d) Image of the second turbo [29].

correct the migration through the range cells (MTRC) of fast rotating targets. The proposed algorithm applies the keystone transform on segments of the data before performing the image formation. The technique was shown to be effective on simulated data achieving good computational efficiency with an increase in the number of segments used to divide the data. In addition the algorithm tested on simulated data provided high resolution and robustness with respect to the noise.

In [31], two techniques for separation of the rigid part from rotating parts were proposed. The first used the order statistic technique applied on the time frequency distributions, the second used the Radon transform. Both techniques were shown to provide good results on simulated data. However, the authors stated that the Radon transform based technique is limited and works on very emphatic micro-Doppler cases. The use of the order statistic technique requires an increased computational load that was not quantified in the paper. In addition both techniques were tested on simulated data with high signal to clutter ratio, while a more common case is of micro-Doppler signatures embedded in strong clutter.

In [32], an alternative view of $\mathrm{m}-\mathrm{D}$ is given. Here the $\mathrm{m}$-D was introduced specifically for ISAR deception Jamming in order to reduce the efficiency of target recognition techniques. In the method proposed in [32] the intercepted radar signal is modulated by a template of false targets. The proposed method requires the interception of the radar pulse transmitted by the ISAR and extraction of the phase informations. Then an image synthesis system produces the required modulation for the false-target and the false-echo is transmitted to the ISAR. In Figure 14a,b the resulting ISAR images without and with a $3 \mathrm{~dB}$ jamming are shown. The target is still visible in the focussed image. However, its recognition would be more difficult because the ghost echoes would lead a target recognition system to identify the target as an airplane with a turbine. The technique proposed in [32] is interesting but further analysis and experiments will be probably required to prove its usefulness.

The presented papers demonstrate how the information coming from the micro-motions of a target can affect the return to a SAR/BSAR/ISAR platform. This family of radar systems provide the advantage to analyse the target reflectivity image of the target jointly with its micro-motion features. The radar imaging community could then take advantage of this complementary source of information for example to remove uncertainty in automatic target recognition systems. 

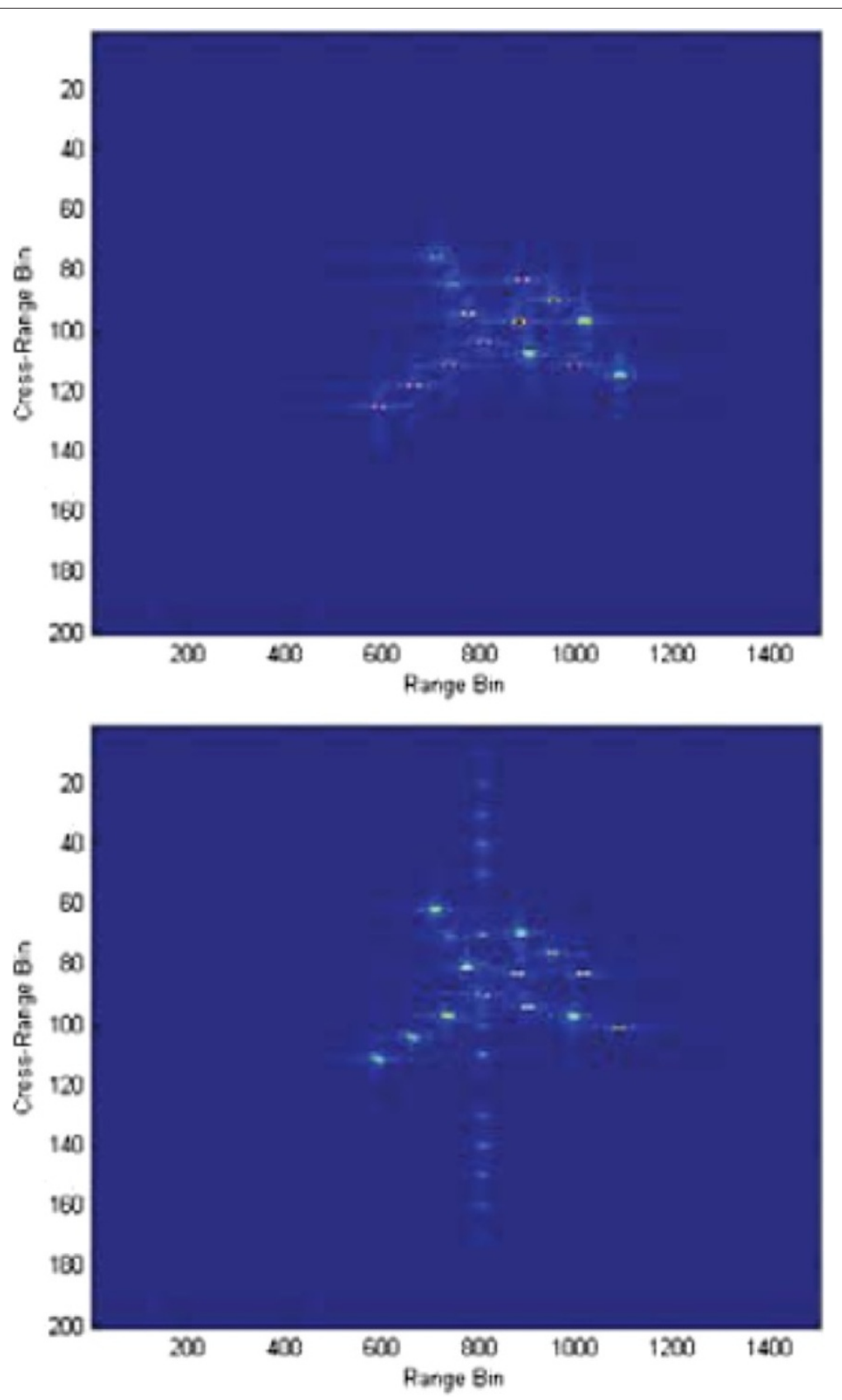

Figure 14 ISAR image: (Top) without jamming, (Bottom) with micro-motion jamming [32].

\subsection{Ultrasound micro-Doppler}

In the last decade there has been a growing interest in applying $\mathrm{m}-\mathrm{D}$ techniques to air acoustic sensors operating at ultrasound frequencies. In particular the literature shows a number of investigations where ultrasound $\mathrm{m}-\mathrm{D}$ target signatures have been used to detect and classify humans and animals with operating frequencies ranging from $20 \mathrm{kHz}$ up to around $80 \mathrm{kHz}$. There are a number of reasons for this; firstly the electromagnetic spectrum is becoming over-congested and hence the use of acoustics can become a valid substitute in situation where long range detection is not a tight requirement. Second the hardware, including the transducers and the electronics, is on the average much cheaper than its radar counter part although, recently, a range of low-power and cost effective super-etherodyne radars have become available off-the-shelf. In particular, for a much lower price, it is possible to operate at frequencies that corresponds to small wavelengths and hence high Doppler shifts; for example the Doppler shift due to a target that moves with 
a speed of $10 \mathrm{~m} / \mathrm{s}$ at $40 \mathrm{kHz}$ in the ultrasound regime is the same as that achieved by a radar operating at $34 \mathrm{GHz}$. On the other hand the use of acoustic sensors has a significant drawback compared to RF systems. The attenuation of acoustic pressure waves in air increases dramatically with frequency and the maximum operating range is often limited to a few tens of metres.

In [33], Zhang et al. present a portable acoustic m-D radar system with an operational frequency of $40 \mathrm{kHz}$ and report the results of a set of measurements in which human gait signatures were acquired indoor and outdoor. The system was composed of two transducers, one transmitting loudspeaker and one receiving microphone, followed by an amplification chain and a data acquisition card that was used to sample data at a rate of $1 \mathrm{MHz}$. During the experiments, the system was placed at human knees height. The experiment demonstrated that collection of $\mathrm{m}$-D signatures of humans at these range of frequency is feasible (Figure 15).

This experiment was repeated in [34] to gather m-D signatures of a) a $9.5 \mathrm{lb}$ Miniature Pinscher in a 30 feet long indoor corridor, b) an American Pitbull and a horse in a farm and c) two personnel targets walking together in a parking lot. The experimental set up was the same of [33] but an accelerometer was placed on the humans' ankle to verify the $\mathrm{m}$-D signatures. The authors showed that the signatures of these four-legged animals are significantly different to those of humans.
A low cost (about 20 USD) acoustic Doppler sensor (ADS) made of off-the-shelf components was developed in [35] to collect $m-D$ signatures of personnel targets at $40 \mathrm{kHz} . \mathrm{m}-\mathrm{D}$ signatures of humans walking towards and away from the sensor were collected with the ADS positioned at about waist height. A small loudspeaker, with diameter as small as 1 wavelength $(8.6 \mathrm{~mm})$, was used to achieve a sonar beamwidth of about 60 degrees. The Doppler signatures were then used to extract Cepstral features to feed a Bayesian classifier modelled with Gaussian mixtures. Experiments were repeated for different locations and set ups with about 30 targets (males and females). Classification performance was assessed between, targets walking away vs target walking towards the sensor, male vs female and identification performance was also assessed. It is interesting to observe that in this paper the information contained in the main Doppler shift of main bulk of the targets is used to perform classification.

Following this work, Zhang et al. [36] significantly reduced their $1 \mathrm{MHz}$ sampling rate of [33] by deploying a simple and cost-effective bandpass sampling approach. They repeated their experiments with the modified system and gathered m-D signatures of eight humans, five males and three females, with the new system and the old system simultaneously. The signatures were used to extract Cepstral features from the data and to perform classification with a Bayesian classifier based on Gaussian

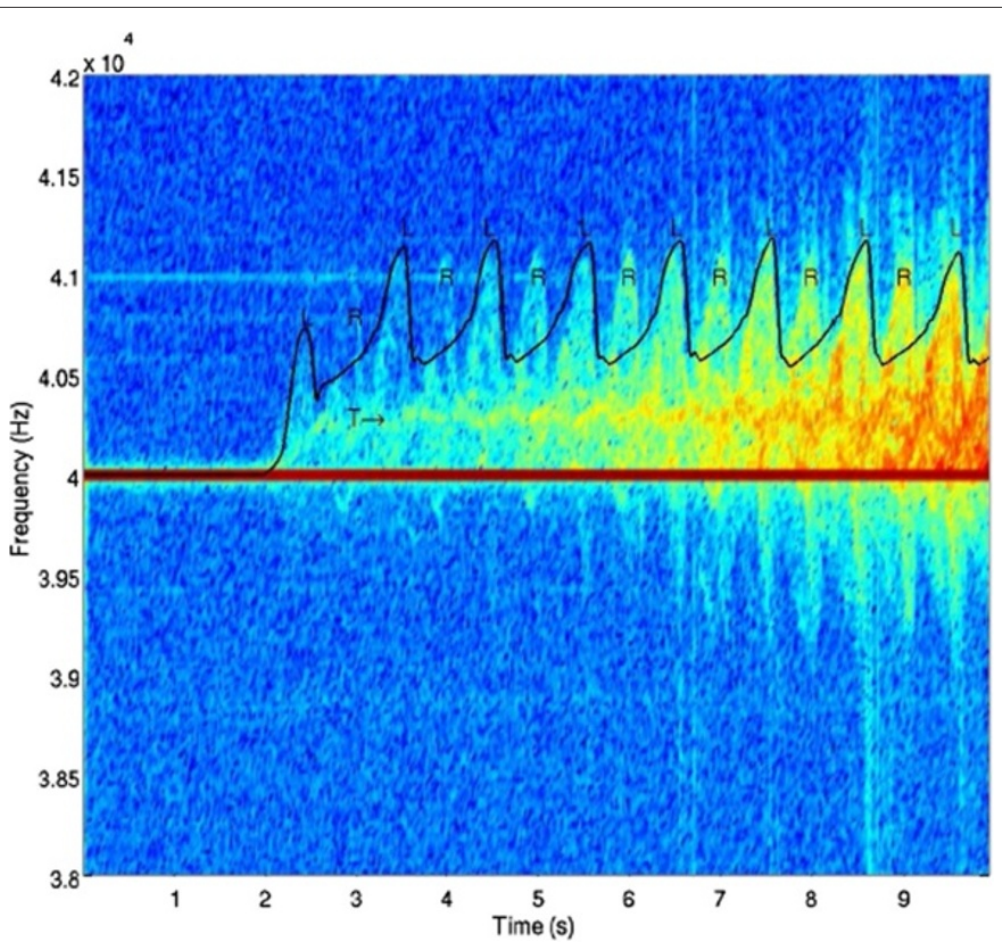

Figure $15 \mathrm{~m}$-D signature of a person walking towards the ultrasound radar as reported in [33]. 
mixture models. Classification results are presented and performance is compared to that obtained in [35].

In [37], Mehmood et al. study the acoustic m-D signatures of single parts of the human body and analyse their contributions. This was achieved by deploying a card-board acoustic screen to isolate the human parts of interest. Data was collected at $40 \mathrm{kHz}$ for personnel targets walking on a treadmill and the height of the sensor was adjusted each time so to face the body component to ensonify. Results are compared to a model developed by Bradley in [38] and differences and similarities are discussed. An example of the signatures of the leg and the torso of a personnel target is given in Figure 16.

Another $40 \mathrm{kHz}$ ultrasound sensor for collection of $\mathrm{m}$ D signatures of personnel targets is presented in [39]. As in previous work, this is a low cost, compact system characterised by a low processing power. This system was deployed in a variety of experiments aiming at demonstrate that $\mathrm{m}-\mathrm{D}$ signatures can be successfully used to classify between different human actions. The experiments were carried out for different geometries and with targets moving on and off a treadmill. The authors showed that classification performance of a $k$-Nearest-Neighbour classifier reached values over $95 \%$ average correct classification when $50 \%$ of the signatures were randomly selected to form the training set. The same sensor was used in [40] to investigate classification performance of a $k-\mathrm{NN}$ classifier as a function of what the authors named "clusters", for recognition of gender and for biometric. An experimental trial aiming at collecting multimodal acoustic data including $\mathrm{m}-\mathrm{D}$ signatures is described in [41].

Following these works, Balleri et al. [42,43] exploited the feasibility of $\mathrm{m}-\mathrm{D}$ techniques at higher frequency so to move to operational frequencies over the hearing sensitivity of most pets and to allow higher Doppler spreads. In [42], a non-coherent and frequency agile ultrasound radar for collection of $\mathrm{m}-\mathrm{D}$ signatures is presented. Here, an acoustic camera was deployed to gather target reflection from two personnel targets and a propeller when tones limited to 20 and $40 \mathrm{kHz}$ were transmitted with a loudspeaker. The acoustic camera is a sensor designed for collection of acoustic data in the audible domain and did not allow recording at higher frequencies. The system was significantly improved in [43], by replacing the acoustic camera with a suitable microphone, to allow operations at $80 \mathrm{kHz}$. A set of experiments was carried out to gather $\mathrm{m}$-D signatures of personnel targets undertaking different action and typical feature extraction algorithms and classifier were used to assess classification performance. Because the sensor was arranged at waist height and because at such high frequency the loudspeaker had a very narrow beamwidth it can be observed that in this work the m-D signatures due the arms of the targets contribute much higher Doppler. An example of the signature of a personnel target walking with an object in a hand is given in Figure 17.

The papers presented here show that ultrasound sensors could represent a cost effective alternative to radar systems for applications that do not require long range detection. They certainly demonstrate that target detection and classification by ultrasound m-D signatures is achievable and they might potentially open up a new research avenue looking at the exploitation of these techniques in future systems.

\subsection{The use of micro-Doppler in through-the-wall applications}

Through-the-wall applications represent another area where the use of $\mathrm{m}$-D signatures for target classification may provide a significant breakthrough. Indeed, increasing research efforts have been dedicated to assessing the impact of through-the-wall propagation on $\mathrm{m}$-D signatures in the last years and this is because, in the last decade, the ability to detect, track and classify moving targets behind walls has been become imperative to address a)

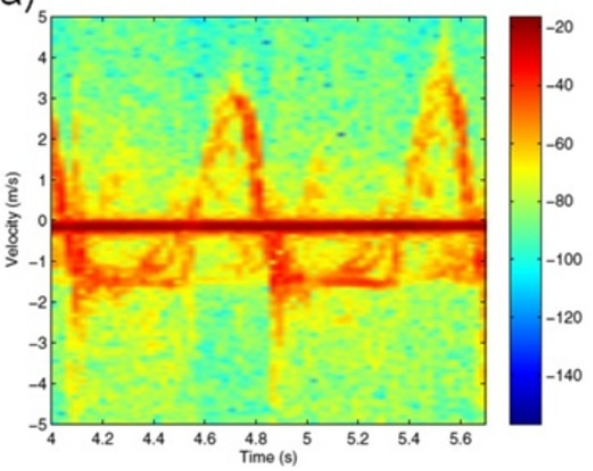

b)

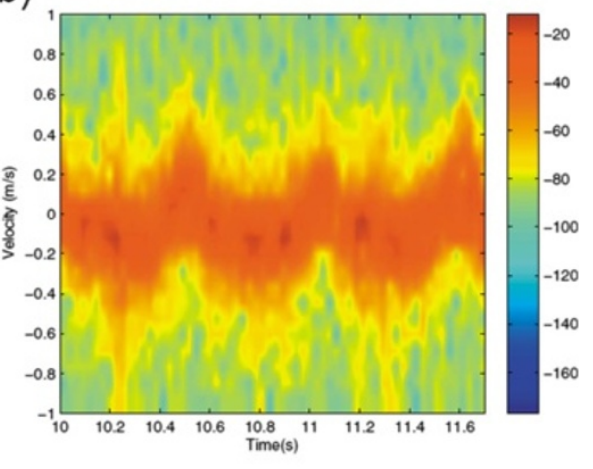

Figure $16 \mathrm{~m}$-D signature of (a) the leg and (b) the torso of a person walking on treadmill. Taken from [37]. 


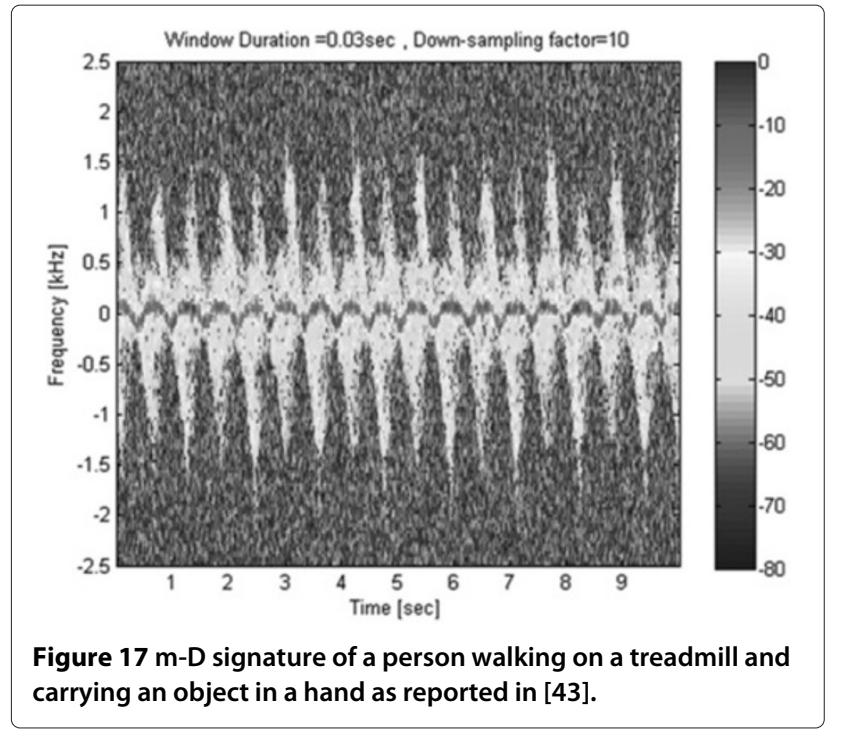

many security and safety issues. Some example are applications such as non-invasive indoor surveillance, searchand-rescue missions, anti terrorism, urban warfare and law enforcement.

In [44], Liu et al. recognise that through-the-wall SAR imaging significantly deteriorates when targets are characterised by a non uniform motion. They exploited the effect of through-the-wall propagation on human m-D signatures and showed that in the presence of homogeneous non-magnetic walls the quality and the properties of $\mathrm{m}$-D signatures remained substantially unaltered and, nevertheless, much more unaffected than SAR images. These results were corroborated by Ram et al. in [45]. Here the authors present a simulation tool that generates $\mathrm{m}$-D signatures of humans behind a wall and they investigate the effect of propagation through homogeneous and non-homogeneous walls on the signatures. The authors here validated their results with real data which was collected with a $2.4 \mathrm{GHz}$ Doppler radar and showed that the presence of the walls does not significantly alter $\mathrm{m}-\mathrm{D}$ signatures. Figure 18 shows a comparison between the measured m-D signature of a human walking in free space and those of the same target walking behind a 15 inch wall and a 12 inch cinderblock wall.

In [46], Chen et al. present a through-the-wall noise radar that combines target detection, target imaging and $\mathrm{m}$-D capabilities. Here the authors present the results of an experimental trial that was carried out to collect m-D signatures of moving humans behind walls performing a number of activities which included arm waving and breathing. Data was gathered for targets moving a) behind a wall made of $10 \mathrm{~cm}$-thick concrete blocks and 2) behind a plastic shed. Results demonstrate promising capabilities for classification of human activities by through-the-wall $\mathrm{m}$-D signatures.

Micro Doppler signatures have also been studied to quantify the undesired errors induced by movement on the estimation of the position of human targets behind walls [47]. Also m-D signatures have been used to detect and classify humans around corners [48]. These techniques, however, rely on multipath propagation and hence on wall reflections rather than through- the-wall propagation.

The presented papers shows the potential of the through wall $\mathrm{m}$-D signatures for target detection and classification. Much more work is expected to be done in order to quantify the performances. For example studies to be carried on should deal with different system bandwidths, carrier frequencies, type of wall and scenarios behind the wall. This is still an early stage for this research but the impact and benefits that can be achieved are significant.

\section{Conclusion}

We have presented a review of the work published in the last 10 years on radar $\mathrm{m}$-D target classification using SAR and ISAR systems as well as the use of this method
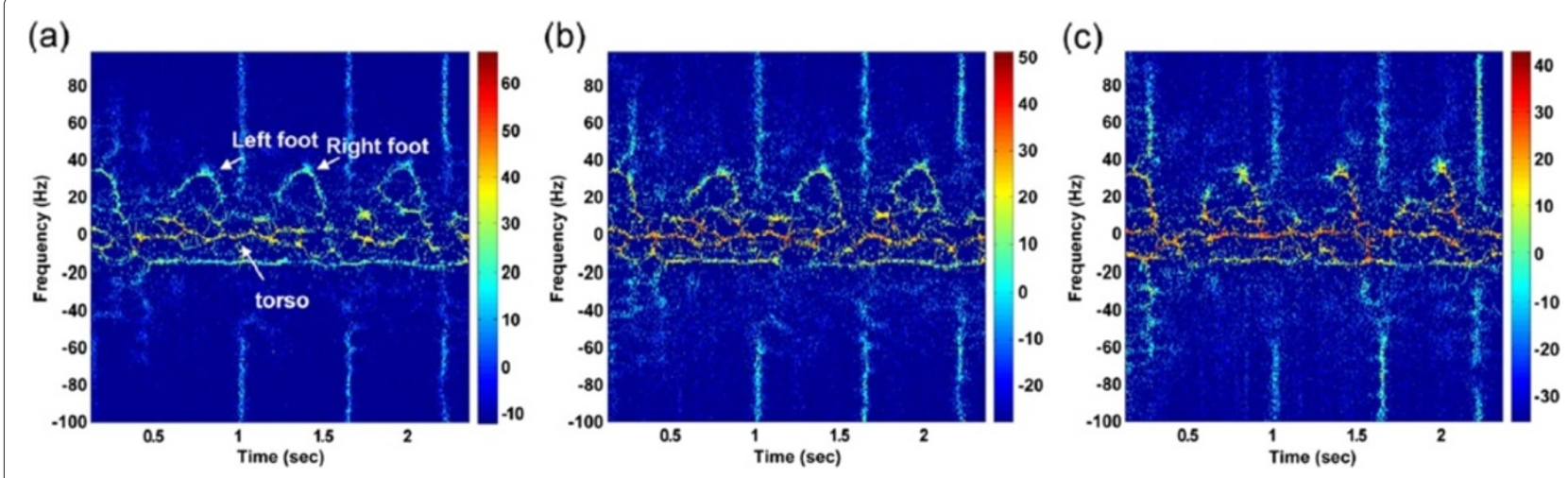

Figure 18 Micro-Doppler signature of a human walking (a) in free space, (b) behind a 15 inch wall and (c) behind a 12 inch cinderblock wall. Taken from [45]. 
in key emerging radar applications such as bistatic SAR, through-the-wall radars and ultrasound radars.

The research on imaging radars demonstrated that the $m-D$ features presented in the return can be used for target classification with the potential to be integrated into existing automatic target recognition systems and improve target classification capabilities.

The research on ultrasound radars presented here demonstrated that target detection and classification by ultrasound micro-Doppler signatures is achievable and showed that ultrasound sensors could represent a cost effective alternative to radar systems for applications that do not require long range detection. Indeed, they might potentially open up a new research avenue looking at the exploitation of these techniques in future systems.

The review of through-the-wall research activities indicated that m-D signatures may not be significantly affected by through-the wall propagation. This represents a first attempt to tackle the problem of detection and classification of targets behind walls with $\mathrm{m}-\mathrm{D}$ signatures and much more work is expected to quantify performance. This research showed that potentially there are significant advantages and benefits of using $\mathrm{m}-\mathrm{D}$ techniques for this application.

Overall research into $\mathrm{m}-\mathrm{D}$ is highly active and fast moving and demonstrated that $\mathrm{m}-\mathrm{D}$ techniques can provide important solutions to many radar target classification challenges.

\section{Competing interests}

The authors declare that they have no competing interests.

\section{Acknowledgements}

This study was supported by the Engineering and Physical Sciences Research Council of the United Kingdom (grants N. EP/H012877/1 and EP/H01 1625/1), the UK MoD University Defence Research Centre in Signal Processing and Selex-Galileo Edinburgh.

\section{Author details}

${ }^{1}$ CeSIP, Department of Electronic and Electrical Engineering, University Of Strathclyde, Glasgow, G1 1XW, UK. ${ }^{2}$ Informatics and Systems Engineering, Cranfield University, Defence Academy of the UK, Shrivenham, SN6 8LA, UK. ${ }^{3}$ Department of Electronic \& Electrical Engineering, University College London, London, WC1E 7JE, UK.

Received: 31 July 2012 Accepted: 9 February 2013

Published: 12 March 2013

\section{References}

1. VChen, $\mathrm{FLi}, \mathrm{SS} \mathrm{Ho}, \mathrm{H}$ Wechsler, Micro-Doppler effect in radar: phenomenon, model, and simulation study. IEEE Trans. Aerosp. Electron. Syst. 42, 2-21 (2006)

2. V Chen, Micro-Doppler Effect in Radar. (Artech House, Norwood, 2011)

3. D Tahmoush, I Silvious, in IEEE 3rd International Conference on Biometrics: Theory, Applications, and Systems, BTAS. Radar micro-doppler for long range front-view gait recognition, (2009), pp. 1-6

4. P Molchanov, J Astola, K Egiazarian, A Totsky, in 2012 IEEE Radar Conference (RADAR). Classification of ground moving radar targets by using joint time-frequency analysis, (2012), pp. 0366-0371

5. P Molchanov, J Astola, K Egiazarian, A Totsky, in Microwaves, Radar and Remote Sensing Symposium (MRRS). Ground moving target classification by using DCT coefficients extracted from micro-Doppler radar signatures and artificial neuron network, (2011), pp. 173-176

6. PA Molchanov, JT Astola, KO Egiazarian, GI Khlopov, VY Morozov, BB Pospelov, AV Totsky, in 11 th International Radar Symposium (IRS). Object recognition in ground surveillance doppler radar by using bispectrum-based time-frequency distributions, (2010), pp. 1-4

7. P Molchanov, J Astola, K Egiazarian, A Totsky, in 2011 European Radar Conference (EURAD). Moving target classification in ground surveillance radar ATR system by using novel bicepstral-based information features, (2011), pp. 194-197

8. S Bjorklund, T Johansson, H Petersson, in 2012 IEEE Radar Conference (RADAR). Evaluation of a micro-Doppler classification method on mm-wave data, (2012), pp. 0934-0939

9. GE Smith, K Woodbridge, CJ BAKER, in The Institution of Engineering and Technology Seminar on High Resolution Imaging and Target Classification. Template based micro-Doppler signature classification, (2006), pp. 127-144

10. G Smith, K Woodbridge, C Baker, H Griffiths, Multistatic micro-Doppler radar signatures of personnel targets. IET Signal Process. 4(3), 224-233 (2010)

11. GE Smith, K Woodbridge, CJ Baker, Radar micro-Doppler signature classification using dynamic time warping. IEEE Trans. Aerosp. Electron. Syst. 46(3), 1078-1096 (2010)

12. G Smith, K Woodbridge, C Baker, in RADAR'08. IEEE Radar Conference. Multistatic micro-Doppler signature of personnel, (2008), pp. 1-6

13. L Novak, G Owirka, W Brower, in Conference Record of the Thirty-Second Asilomar Conference o Signals, Systems \& Computers, November 1-4 1998, vol. 1. An efficient multi-target SAR ATR algorithm (Pacific Grove California, 1998), pp. 3-13

14. M Ruegg, E Meier, D Nuesch, Vibration and rotation in millimeter-wave SAR. IEEE Trans. Geosci. Rem. Sens. 45(2), 293-304 (2007)

15. T Sparr, B Krane, Micro-Doppler analysis of vibrating targets in SAR. IEE Proc. Radar Sonar Navig. 150(4), 277-83 (2003)

16. S Silverstein, C Hawkins, in Conference Record of the Thirty-Eighth Asilomar Conference on Signals, Systems and Computers, November 7-10, 2004, vol. 2. Synthetic aperture radar image signatures of rotating objects (Monterey CA, 2004), pp. 1663-1667

17. T Sparr, in 2005 IEEE International Radar Conference. Moving target motion estimation and focusing in SAR images, (2005), pp. 290-294

18. $X \mathrm{Li}, \mathrm{B}$ Deng, $Y$ Qin, $\mathrm{H}$ Wang, $Y \mathrm{Li}$, The influence of target micromotion on SAR and GMTI. IEEE Trans. Geosci. Rem. Sens. 49(7), 2738-2751 (2011)

19. C Clemente, JJ Soraghan, in 9th European Conference on Synthetic Aperture Radar, EUSAR. Vibrating micro-Doppler signature extraction from SAR data using singular value decomposition, (2012), pp. 191-194

20. C Clemente, JJ Soraghan, Vibrating target micro-Doppler signature in bistatic SAR with a fixed receiver. IEEE Trans. Geosci. Rem. Sens. 50(8), 1-9 (2012)

21. W Zhang, C Tong, Q Zhang, Y Zhang, X Zhang, Extraction of vibrating features with dual-channel fixed-receiver bistatic SAR. IEEE Geosci. Rem. Sens. Lett. 9(3), 507-511 (2012)

22. A Ghaleb, L Vignaud, J Nicolas, Micro-Doppler analysis of wheels and pedestrians in ISAR imaging. IET Signal Process. 2(3), 301-311 (2008)

23. VChen, W Miceli, B Himed, in International Radar Conference-Surveillance for a Safer World. Micro-Doppler analysis in ISAR-review and perspectives, (2009), pp. 1-6

24. L Bin, W Jian-wei, Y Kang-ze, W Yan, C Lin-lin, L Jun, in 1st Asian and Pacific Conference on Synthetic Aperture Radar, 2007. APSAR. ISAR based on micro-doppler analysis and Chirplet parameter separation, (2007), pp. 379-384

25. S Fulin, J Mingyuan. 8th European Conference on Synthetic Aperture Radar (EUSAR). ISAR imaging of target with micro-motion parts based on SSA, (2010), pp. 1-4

26. F Zhu, Y Luo, Q Zhang, YQ Feng, YQ Bai, ISAR imaging for Avian species identification with frequency-stepped chirp signals. IEEE Geosci. Rem. Sens. Lett. 7, 151-155 (2010)

27. T Thayaparan, L Stankovic, P Suresh, KVenkataramaniah, K SivaSankaraSai, T Sairam, S Shankar, K Nikhilesh, in 11th International Radar Symposium (IRS). Focusing ISAR images of moving targets in real-time using time-frequency-based method, (2010), pp. 1-4 
28. X Bai, M Xing, F Zhou, G Lu, Z Bao, Imaging of micromotion targets with rotating parts based on empirical-mode decomposition. IEEE Trans. Geosci. Rem. Sens. 46(11), 3514-3523 (2008)

29. X Bai, F Zhou, M Xing, Z Bao, High resolution ISAR imaging of targets with rotating parts. IEEE Trans. Aerosp. Electron. Syst. 47(4), 2530-2543 (2011)

30. K Huo, Y Liu, J Hu, W Jiang, X Li, A novel imaging method for fast rotating targets based on the segmental Pseudo keystone transform. IEEE Trans. Geosci. Rem. Sens. 49(4), 1464-1472 (2011)

31. L Stankovic, I Djurovic, T Thayaparan, Separation of target rigid body and micro-doppler effects in ISAR imaging. IEEE Trans. Aerosp. Electron. Syst. 42(4), 1496-1506 (2006)

32. Z Ben-yu, X Lei, B Da-ping, in IEEE 10th International Conference on Signal Processing (ICSP). A micro-motion feature deception jamming method to ISAR, (2010), pp. 2287-2290

33. Z Zhang, PO Pouliquen, A Waxman, AG Andreou, Acoustic micro-Doppler radar for human gait imaging. J. Acoust. Soc. Am. 121(3), EL110-EL113 (2007). http://link.aip.org/link/?JAS/121/EL110/1

34. Z Zhang, P Pouliquen, A Waxman, A Andreou, in CISS '07. 41st Annual Conference on Information Sciences and Systems. Acoustic micro-Doppler gait signatures of humans and animals, (2007), pp. 627-630

35. K Kalgaonkar, B Raj, in IEEE Conference on Advanced Video and Signal Based Surveillance, 2007 AVSS. Acoustic Doppler sonar for gait recogination, (2007), pp. 27-32

36. Z Zhang, A Andreou, in Argentine School of Micro-Nanoelectronics, Technology and Applications, 2008 EAMTA. Human identification experiments using acoustic micro-Doppler signatures, (2008), pp. 81-86

37. A Mehmood, JM Sabatier, M Bradley, A Ekimov, Extraction of the velocity of walking human's body segments using ultrasonic Doppler. J. Acoust. Soc. Am. 128(5), EL316-EL322 (2010). http://link.aip.org/link/?JAS/ 128/EL316/1

38. M Bradley, JM Sabatier, Applications of Fresnel-Kirchhoff diffraction theory in the analysis of human-motion Doppler sonar grams. J. Acoust. Soc. Am. 128(5), EL248-EL253 (2010). http://link.aip.org/link/?JAS/ 128/EL248/1

39. S Dura-Bernal, G Garreau, C Andreou, A Andreou, J Georgiou, T Wennekers, S Denham, in Proceedings of the Second international conference on Human Behavior Unterstanding HBU'11. Human action categorization using ultrasound micro-doppler signatures (Springer-Verlag Berlin, Heidelberg, 2011), pp. 18-28

40. G Garreau, C Andreou, A Andreou, J Georgiou, S Dura-Bernal, T Wennekers, S Denham, in 2011 IEEE Biomedical Circuits and Systems Conference (BioCAS). Gait-based person and gender recognition using micro-doppler signatures, (2011), pp. 444-447

41. J Georgiou, P Pouliquen, A Cassidy, G Garreau, C Andreou, G Stuarts, C d'Urbal, A Andreou, S Denham, T Wennekers, R Mill, I Winkler, T Bohm, O Szalardy, G Klump, S Jones, A Bendixen, in 45th Annual Conference on Information Sciences and Systems (CISS). A multimodal-corpus data collection system for cognitive acoustic scene analysis, (2011), pp. 1-6

42. A Balleri, K Woodbridge, K Chetty, in 2011 IEEE Radar Conference. Frequency-agile non-coherent ultrasound radar for collection of micro-Doppler signatures, (2011), pp. 45-48

43. A Balleri, K Chetty, K Woodbridge, Classification of personnel targets by acoustic micro-doppler signatures. IET Radar Sonar Navig. 5(9), 943-951 (2011)

44. X Liu, H Leung, G Lampropoulos, Effects of non-uniform motion in through-the-wall SAR imaging. IEEE Trans. Antennas Propag. 57(11), 3539-3548 (2009)

45. S Ram, C Christianson, Y Kim, H Ling, Simulation and analysis of human Micro-Dopplers in through-wall environments. IEEE Trans. Geosci. Rem. Sens. 48(4), 2015-2023 (2010)

46. PH Chen, MC Shastry, CP Lai, RM Narayanan, A portable real-time digital noise radar system for through-the-wall imaging. IEEE Trans. Geosci. Rem. Sens. PP(99), 1-12 (2012)
47. P Setlur, M Amin, F Ahmad, Dual-frequency Doppler radars for indoor range estimation: Cramer-Rao bound analysis. IET Signal Process. 4(3), 256-271 (2010)

48. A Sume, M Gustafsson, M Herberthson, A Janis, S Nilsson, J Rahm, A Orbom, Radar detection of moving targets behind corners. IEEE Trans. Geosci. Rem. Sens. 49(6), 2259-2267 (2011)

doi:10.1186/1687-6180-2013-47

Cite this article as: Clemente et al:: Developments in target micro-Doppler signatures analysis: radar imaging, ultrasound and through-the-wall radar. EURASIP Journal on Advances in Signal Processing 2013 2013:47.

\section{Submit your manuscript to a SpringerOpen ${ }^{\mathcal{O}}$ journal and benefit from:}

- Convenient online submission

- Rigorous peer review

- Immediate publication on acceptance

- Open access: articles freely available online

- High visibility within the field

- Retaining the copyright to your article

Submit your next manuscript at $\gg$ springeropen.com 